\title{
Efficient Syntheses of KDR Kinase Inhibitors Using a Pd-Catalyzed Tandem C-N/Suzuki Coupling as the Key Step
}

\section{Table of Content}

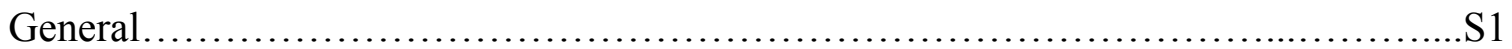

2-Nitro-5-(2-piperidin-1-ylethoxy)benzaldehyde (26b) ............................. 2

1-\{2-[3-(2,2-Dibromovinyl)-4-nitrophenoxy]-ethyl $\}$-piperidine (28b)..................S3

2-(2,2-Dibromovinyl)-4-(2-piperidin-1-ylethoxy)phenylamine (29b) ..................S3

2-Methoxy-3-[5-(2-piperidin-1-ylethoxy)-1H-indol-2-yl]quinoline (30b) ................S4

3-[5-(2-Piperidin-1-ylethoxy)-1H-indol-2-yl]-1H-quinolin-2-one (3) ....................S4

5-\{2-[(2-Methoxyethyl)-methylamino]-ethoxy $\}$-2-nitrobenzaldehyde (26c)............S5

$\{2-[3-(2,2-D i b r o m o v i n y l)-4-n i t r o p h e n o x y]-e t h y l\}-(2-m e t h o x y e t h y l)-m e t h y l a m i n e \quad(28 c)$ ..S6

2-(2,2-Dibromovinyl)-4-\{2-[(2-methoxyethyl)-methylamino]-ethoxy $\}$-phenylamine (29c)

(2-Methoxyethyl)-\{2-[2-(2-methoxyquinolin-3-yl)-1H-indol-5-yloxy]-ethyl $\}$ methylamine (30c) S7

3-(5-\{2-[(2-Methoxyethyl)-methylamino]-ethoxy $\}-1 \mathrm{H}-$ indol-2-yl)-1H-quinolin-2-one (4)

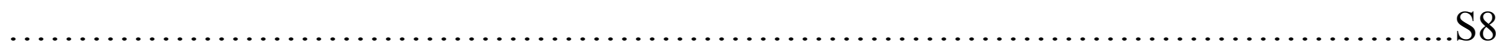

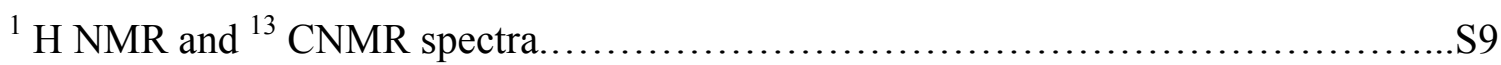

\section{General}

All flasks were flame-dried under a stream of nitrogen or argon and cooled before use unless otherwise noted. Solvents and solutions were transferred with syringes or cannulae using standard inert atmosphere techniques.

${ }^{1} \mathrm{H}$ and ${ }^{13} \mathrm{C}$ NMR spectra were obtained using a Varian Mercury-300, Gemini-300, Unity-400, Mercury 400, or Unity-500 spectrometer. ${ }^{1} \mathrm{H}$ NMR spectra were referenced to tetramethylsilane (TMS, $0 \mathrm{ppm})$ using $\mathrm{CDCl}_{3}$ as solvent, DMSO- $\mathrm{D}_{5}$ residue peaks $(2.50$ ppm) using DMSO-d $\mathrm{d}_{6}$ as solvent. ${ }^{13} \mathrm{C}$ NMR spectra were referenced to solvent carbons (77.23 ppm for $\mathrm{CDCl}_{3} ; 39.57 \mathrm{ppm}$ for $\left.\mathrm{DMSO}-\mathrm{d}_{6}\right)$. When carbons are equivalent, no special notation is used. 
Melting points were taken on a Fisher-Johns melting point apparatus without correction. IR spectra were obtained using a Nicolet DX FT IR spectrometer using thin films of products coated on $\mathrm{NaCl}$ plates. Only absorption frequencies higher than 1000 $\mathrm{cm}^{-1}$ are reported. High-resolution mass spectra were obtained from a VG 70-250S (double focusing) mass spectrometer at $70 \mathrm{eV}$.

Analytical TLC was performed using EM Separations pre-coated silica gel $0.2 \mathrm{~mm}$ layer UV 254 fluorescent sheets. Column chromatography was carried out as "flash chromatography" as reported by Still on Ultra Pure Silica Gel (Silicycles, 230-400 mesh, $60 \mathrm{~A})$ silica gel using the indicated eluent. ${ }^{1}$ Unless otherwise specified, extracts were dried over $\mathrm{MgSO}_{4}$ and volatile solvents were removed with a rotary evaporator at aspirator pressure.

\section{2-Nitro-5-(2-piperidin-1-ylethoxy)benzaldehyde (26b)}<smiles>O=Cc1cc(O)ccc1[N+](=O)[O-]</smiles><smiles>O=Cc1cc(OCCN2CCCCC2)ccc1[N+](=O)[O-]</smiles>

To a 10-mL round-bottomed flask was charged with 5-hydroxy-2-nitrobenzaldhyde (0.501 g, $3 \mathrm{mmol})$, 1-(2-chloroethyl)piperidine hydrochloride (0.828 g, $4.5 \mathrm{mmol})$, and $\mathrm{K}_{2} \mathrm{CO}_{3}(1.25 \mathrm{~g}, 9 \mathrm{mmol})$. After purged with argon for $5 \mathrm{~min}$, anhydrous DMF (3 mL) was added and the mixture was heated at $45^{\circ} \mathrm{C}$ overnight $(16 \mathrm{~h})$. The mixture was poured into $1 \mathrm{M} \mathrm{NaOH}(5 \mathrm{~mL})$, extracted with $\mathrm{Et}_{2} \mathrm{O}(3 \times 20 \mathrm{~mL})$, washed with $\mathrm{NaHCO}_{3}(10 \mathrm{~mL})$, $\mathrm{H}_{2} \mathrm{O}(10 \mathrm{~mL})$, brine $(10 \mathrm{~mL})$, and dried over $\mathrm{Na}_{2} \mathrm{SO}_{4}$. The crude material was purified using flash chromatography $\left(3 \% \mathrm{Et}_{3} \mathrm{~N}, 32 \% \mathrm{EtOAc}\right.$ and $65 \%$ hexanes) to afford the product was a slight yellow oil $(0.710 \mathrm{~g}, 85 \%) .{ }^{1} \mathrm{H}$ NMR (400 MHz, $\left.\mathrm{CDCl}_{3}\right) \delta 10.48(1 \mathrm{H}$, s), $8.15(1 \mathrm{H}, \mathrm{d}, J=9.0 \mathrm{~Hz}), 7.34(1 \mathrm{H}, \mathrm{d}, J=2.9 \mathrm{~Hz}), 7.16(1 \mathrm{H}, \mathrm{dd}, J=9.0,2.9 \mathrm{~Hz}), 4.23$ $(2 \mathrm{H}, \mathrm{t}, J=5.8 \mathrm{~Hz}), 2.81(2 \mathrm{H}, \mathrm{t}, J=5.9 \mathrm{~Hz}), 2.50(4 \mathrm{H}, \mathrm{t}, J=5.1 \mathrm{~Hz}), 1.61$ (4H, quintet, $J$ $=5.7 \mathrm{~Hz}), 1.45(2 \mathrm{H}$, quintet, $J=5.6 \mathrm{~Hz})$.

\footnotetext{
${ }^{1}$ Still, W. C.; Kahn, M.; Mitra, A. J. Org. Chem. 1978, 43, 2923.
} 


\section{1-\{2-[3-(2,2-Dibromovinyl)-4-nitrophenoxy]-ethyl\}-piperidine (28b)}<smiles>CC(C)CC1(C)CCCCC1</smiles>

To a solution of the aldehyde $(0.174 \mathrm{~g}, 0.625 \mathrm{mmol})$ and $\mathrm{CBr}_{4}(0.311 \mathrm{~g}, 0.94 \mathrm{mmol})$ in DCM (3 mL) was added a solution of $\mathrm{PPh}_{3}(0.493 \mathrm{~g}, 1.88 \mathrm{mmol})$ in DCM $(1 \mathrm{~mL})$ at 0 ${ }^{\circ} \mathrm{C}$. The mixture was stirred for $30 \mathrm{~min}$, warmed to $\mathrm{rt}$, and quenched with $\mathrm{NaHCO}_{3}(5$ $\mathrm{mL})$. The mixture was extracted with $\mathrm{Et}_{2} \mathrm{O}(15 \mathrm{~mL})$ and EtOAc $(15 \mathrm{~mL})$, washed with brine, and dried over $\mathrm{Na}_{2} \mathrm{SO}_{4}$. The crude material was purified by flash chromatography to afford $28 \mathbf{b}$ as a slightly yellow oil $(0.2156 \mathrm{~g}, 79 \%) .{ }^{1} \mathrm{H}$ NMR $\left(500 \mathrm{MHz}, \mathrm{CDCl}_{3}\right) \delta$ $8.15(1 \mathrm{H}, \mathrm{d}, J=9.1 \mathrm{~Hz}), 7.84(1 \mathrm{H}, \mathrm{d}, J=0.5 \mathrm{~Hz}), 7.02(1 \mathrm{H}, \mathrm{d}, J=2.7 \mathrm{~Hz}), 6.97(1 \mathrm{H}, J=$ 9.1, $2.7 \mathrm{~Hz}), 4.20(2 \mathrm{H}, \mathrm{t}, J=7.5 \mathrm{~Hz}), 2.80(2 \mathrm{H}, \mathrm{t}, J=6.0 \mathrm{~Hz}), 2.51(4 \mathrm{H}, \mathrm{t}, J=5.0 \mathrm{~Hz})$, $1.61(4 \mathrm{H}$, quintet, $J=5.7 \mathrm{~Hz}), 1.46(2 \mathrm{H}$, quintet, $J=5.8 \mathrm{~Hz}) .{ }^{13} \mathrm{C}$ NMR $(125 \mathrm{MHz}$, $\left.\mathrm{CDCl}_{3}\right) \delta 162.9,140.1,134.9,134.1,127.6,117.1,115.3,92.8,67.5,57.8,55.4,26.2$, 24.3. HRMS calc'd for $\mathrm{C}_{15} \mathrm{H}_{19} \mathrm{~N}_{2} \mathrm{O}_{3} \mathrm{Br}_{2}\left([\mathrm{M}+\mathrm{H}]^{+}\right)$432.9756. Found: 432.9770 .

\section{2-(2,2-Dibromovinyl)-4-(2-piperidin-1-ylethoxy)phenylamine (29b)}

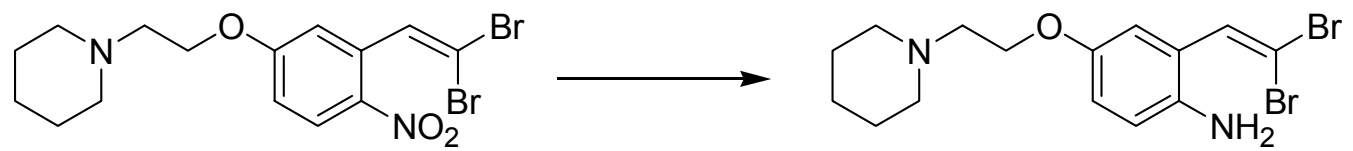

A mixture of the nitrobenzene $(0.215 \mathrm{~g}, 0.495 \mathrm{mmol})$ and $1 \% \mathrm{Pt}-\mathrm{C}[\mathrm{V}](26 \mathrm{mg})$ in $\mathrm{MeOH}(5 \mathrm{~mL})$ was hydrogenated at 25 psi for $3 \mathrm{~h}$ using a Parr hydrogenator. After removal of the catalyst through filtration, the solvent was evaporated to give $\mathbf{2 9 b}$ as a colorless oil (0.189 g, 94\%). IR (neat, $\left.\mathrm{cm}^{-1}\right)$ : 3431, 3361, 2933, 1611, 1497, 1262, 1222 , 1164, 1040. ${ }^{1} \mathrm{H}$ NMR (400 MHz, $\left.\mathrm{CDCl}_{3}\right) \delta 7.33(1 \mathrm{H}, \mathrm{s}), 6.91(1 \mathrm{H}, \mathrm{d}, J=2.9 \mathrm{~Hz}), 6.78$ $(1 \mathrm{H}, \mathrm{dd}, J=8.6,2.9 \mathrm{~Hz}), 6.65(1 \mathrm{H}, \mathrm{d}, J=8.6 \mathrm{~Hz}), 4.08(2 \mathrm{H}, \mathrm{t}, J=5.9 \mathrm{~Hz}), 3.49(3 \mathrm{H}, \mathrm{s})$, $3.45(2 \mathrm{H}, \mathrm{br}), 2.80(2 \mathrm{H}, \mathrm{t}, J=5.9 \mathrm{~Hz}), 2.56(4 \mathrm{H}, \mathrm{br}), 1.68-1.62(4 \mathrm{H}, \mathrm{m}), 1.49-1.46(2 \mathrm{H}$, m). ${ }^{13} \mathrm{C} \mathrm{NMR}\left(100 \mathrm{MHz}, \mathrm{CDCl}_{3}\right) \delta 151.6,137.7,134.1,122.8,117.4,117.2,114.9,92.7$, 
66.7, 58.1, 55.2, 26.0, 24.3. HRMS calc'd for $\mathrm{C}_{15} \mathrm{H}_{21} \mathrm{~N}_{2} \mathrm{OBr}_{2}\left([\mathrm{M}+\mathrm{H}]^{+}\right)$403.0015. Found: 403.0031 .

\section{2-Methoxy-3-[5-(2-piperidin-1-ylethoxy)-1H-indol-2-yl]quinoline (30b)}

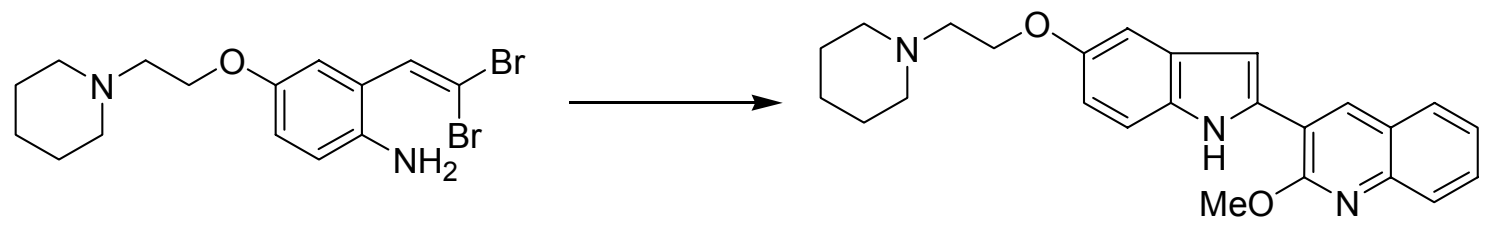

To a $5 \mathrm{~mL}$ round-bottomed flask was charged with aniline $29 \mathrm{~b}(0.0818 \mathrm{~g}, 0.202$ mmol), boronic acid 13 (0.0616 g, $0.30 \mathrm{mmol}), \mathrm{Pd}(\mathrm{OAc})_{2}(2.2 \mathrm{mg}, 0.01 \mathrm{mmol})$, S-Phos (8.2 $\mathrm{mg}, 0.02 \mathrm{mmol})$, and $\mathrm{K}_{3} \mathrm{PO}_{4} \cdot \mathrm{H}_{2} \mathrm{O}(0.184 \mathrm{~g}, 0.8 \mathrm{mmol})$. After the flask was purged with argon for $10 \mathrm{~min}$, PhMe $(5 \mathrm{~mL})$ was added, and the reaction mixture was heated at $100{ }^{\circ} \mathrm{C}$ for $2 \mathrm{~h}$. The reaction mixture was then quenched with $\mathrm{NaHCO}_{3}$, extracted with EtOAc $(3 \times 10 \mathrm{~mL})$, and dried over $\mathrm{Na}_{2} \mathrm{SO}_{4}$. The crude material was purified by flash chromatography using 33\% EtOAc in hexanes containing 3\% $i \mathrm{PrNH}_{2}$ to afford the product 30b as a slightly solid $(0.0531 \mathrm{~g}, 65 \%)$. mp 134-136 ${ }^{\circ} \mathrm{C}$. IR (neat, $\left.\mathrm{cm}^{-1}\right): 3457$, 2935, 1621, 1475, 1249, 1193. ${ }^{1} \mathrm{H}$ NMR (400 MHz, $\left.\mathrm{CDCl}_{3}\right) \delta 9.55(1 \mathrm{H}, \mathrm{br}), 8.41(1 \mathrm{H}, \mathrm{s})$, $7.84(1 \mathrm{H}, \mathrm{dd}, J=7.1,0.8 \mathrm{~Hz}), 7.75(1 \mathrm{H}, \mathrm{dd}, J=7.9,1.3 \mathrm{~Hz}), 7.60(1 \mathrm{H}, \mathrm{ddd}, J=8.2,7.0$, $1.5 \mathrm{~Hz}), 7.40(1 \mathrm{H}, \mathrm{ddd}, J=7.9,7.0,1.3 \mathrm{~Hz}), 7.32(1 \mathrm{H}, \mathrm{d}, J=8.8 \mathrm{~Hz}), 7.11(1 \mathrm{H}, \mathrm{d}, J=2.4$ $\mathrm{Hz}), 6.98(1 \mathrm{H}, \mathrm{dd}, J=2.2,0.9 \mathrm{~Hz}), 6.89(1 \mathrm{H}, \mathrm{dd}, J=8.8,2.4 \mathrm{~Hz}), 4.26(3 \mathrm{H}, \mathrm{s}), 4.18(2 \mathrm{H}$, $\mathrm{t}, J=6.2 \mathrm{~Hz}), 2.81(2 \mathrm{H}, \mathrm{t}, J=6.1 \mathrm{~Hz}), 2.54(4 \mathrm{H}, \mathrm{br}), 1.62(4 \mathrm{H}$, quintet, $J=5.7 \mathrm{~Hz}), 1.45$ $(2 \mathrm{H}$, quintet, $J=6.1 \mathrm{~Hz}) .{ }^{13} \mathrm{C} \mathrm{NMR}\left(100 \mathrm{MHz}, \mathrm{CDCl}_{3}\right) \delta 158.4,153.8,145.3,135.1$, $134.2,132.0,129.6,128.8,127.6,127.1,125.7,125.0,116.9,113.9,112.1,103.1,101.1$, $66.8,58.4,55.3,54.2,26.2,24.5$. HRMS calc'd for $\mathrm{C}_{25} \mathrm{H}_{28} \mathrm{~N}_{3} \mathrm{O}_{2}\left([\mathrm{M}+\mathrm{H}]^{+}\right) 402.2176$. Found: 402.2181 .

\section{3-[5-(2-Piperidin-1-ylethoxy)-1H-indol-2-yl]-1H-quinolin-2-one (3)}




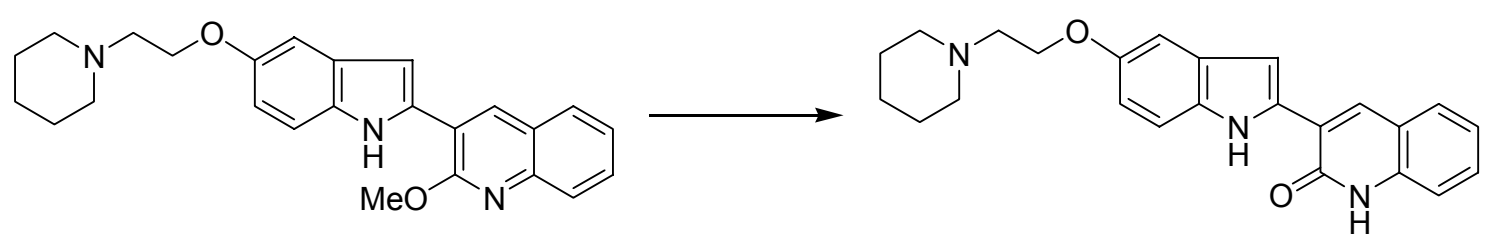

A mixture of $30 b(38 \mathrm{mg}, 0.0946 \mathrm{mmol})$ and $\mathrm{HCl}(3 \mathrm{~mL}, 3 \mathrm{M})$ was heated to $90{ }^{\circ} \mathrm{C}$ overnight. Solid $\mathrm{K}_{2} \mathrm{CO}_{3}$ was carefully added to the mixture until it is neutral, and the mixture was filtered through a Celite pad $(0.2 \mathrm{~g})$. The crude material on celite was washed with sufficient amounts of water and dried under vacuum. The solid was wrapped in a filter paper and loaded in a thimble, and extracted with a Soxlet extractor using a mixed solvent of DCM and $\mathrm{MeOH}$. The resulting solution was concentrated to $\sim 3 \mathrm{~mL}$, and the residual solvent was carefully removed with a pipette. After washed with DCM $(2 \mathrm{~mL})$, the product was dried under vaccum as a yellow solid $(33.6 \mathrm{mg}, 92 \%) .{ }^{1} \mathrm{H}$ NMR $\left(400 \mathrm{MHz}, \mathrm{DMSO}_{-} \mathrm{d}_{6}\right) 11.63(1 \mathrm{H}, \mathrm{s}), 8.51(1 \mathrm{H}, \mathrm{s}), 7.71(1 \mathrm{H}, \mathrm{d}, J=7.7 \mathrm{~Hz}), 7.48(1 \mathrm{H}, \mathrm{t}$, $J=7.4 \mathrm{~Hz}), 7.41(1 \mathrm{H}, \mathrm{d}, J=8.8 \mathrm{~Hz}), 7.37(1 \mathrm{H}, \mathrm{d}, J=8.1 \mathrm{~Hz}), 7.21(1 \mathrm{H}, \mathrm{t}, J=7.5 \mathrm{~Hz})$, $7.19(1 \mathrm{H}, \mathrm{s}), 7.06(1 \mathrm{H}, \mathrm{s}), 6.75(1 \mathrm{H}, \mathrm{dd}, J=8.6,2.1 \mathrm{~Hz}), 4.05(2 \mathrm{H}, \mathrm{t}, J=5.8 \mathrm{~Hz}), 2.66$ $(2 \mathrm{H}, \mathrm{t}, J=5.8 \mathrm{~Hz}), 2.44(4 \mathrm{H}, \mathrm{br}), 1.50(4 \mathrm{H}, \mathrm{m}), 1.38(2 \mathrm{H}, \mathrm{m}) .{ }^{13} \mathrm{C} \mathrm{NMR}(100 \mathrm{MHz}$, $\left.\mathrm{CDCl}_{3}\right) \delta 161.0,152.7,138.1,134.4,133.5,131.7,129.8,128.3,127.7,122.3,122.0$, 119.6, 115.3, 112.8, 112.4, 102.3, 101.4, 66.0, 57.7, 54.5, 25.6, 24.0. HRMS (ESI) calc'd for $\mathrm{C}_{24} \mathrm{H}_{26} \mathrm{~N}_{3} \mathrm{O}_{2}\left([\mathrm{M}+\mathrm{H}]^{+}\right)$388.2019. Found: 388.2023.

\section{5-\{2-[(2-Methoxyethyl)-methylamino]-ethoxy\}-2-nitrobenzaldehyde (26c)}<smiles>O=Cc1cc(O)ccc1[N+](=O)[O-]</smiles><smiles>COCCN(C)CCOc1ccc([N+](=O)[O-])c(C=O)c1</smiles>

(2-Chloroethyl)-(2-methoxyethyl)-methylamine 27c was prepared by neutralization of its hydrochloride, which was prepared according the literature procedure. ${ }^{2}$ To a $10-\mathrm{mL}$ round-bottomed flask was charged with 5-hydroxy-2-nitrobenzaldhyde (1.28 g, 7.6 mmol), 2-chloroethyl)-(2-methoxyethyl)-methylamine (1.51 g, $10 \mathrm{mmol}$ ), and $\mathrm{K}_{2} \mathrm{CO}_{3}$ (2.2 g, $15 \mathrm{mmol})$. After purged with argon for $5 \mathrm{~min}$, anhydrous DMF (5 mL) was added

\footnotetext{
${ }^{2}$ Kuethe, J. T.; Wong, A.; Davies, I. W. Org. Lett. 2003, 5, 3975.
} 
and the mixture was heated at $70{ }^{\circ} \mathrm{C}$ for $6 \mathrm{~h}$. The mixture was poured into $1 \mathrm{M} \mathrm{NaOH}(5$ $\mathrm{mL})$, extracted with $\mathrm{Et}_{2} \mathrm{O}(3 \times 20 \mathrm{~mL})$, washed with $\mathrm{NaHCO}_{3}(10 \mathrm{~mL}), \mathrm{H}_{2} \mathrm{O}(10 \mathrm{~mL})$, brine $(10 \mathrm{~mL})$, and dried over $\mathrm{Na}_{2} \mathrm{SO}_{4}$. The crude material was purified using flash chromatography (5\% $\mathrm{Et}_{3} \mathrm{~N}, 50 \%$ EtOAc and $50 \%$ hexanes) to afford the product was a slight yellow oil (1.01 g, 47\%). IR (neat, $\left.\mathrm{cm}^{-1}\right)$ : 2881, 1694, 1585, 1515, 1332, 1289, 1243, 1119, 1073, 1025. ${ }^{13} \mathrm{C}$ NMR $\left(100 \mathrm{MHz}, \mathrm{CDCl}_{3}\right) \delta 188.7,163.5,142.4,134.5$, 127.4, 119.2, 114.0, 70.7, 67.7, 59.1, 57.4, 56.3, 43.5. HRMS (ESI) calc'd for $\mathrm{C}_{13} \mathrm{H}_{19} \mathrm{~N}_{2} \mathrm{O}_{5}\left([\mathrm{M}+\mathrm{H}]^{+}\right)$283.1288. Found: 283.1283.

\section{\{2-[3-(2,2-Dibromovinyl)-4-nitrophenoxy]-ethyl\}-(2-methoxyethyl)-methylamine} (28c)<smiles>COCCN(C)CCOc1ccc([N+](=O)[O-])c(C=O)c1</smiles>

To a solution of the aldehyde $26 \mathrm{c}(0.384 \mathrm{~g}, 1.36 \mathrm{mmol})$ and $\mathrm{CBr}_{4}(0.681 \mathrm{~g}, 2.05$ mmol) in DCM $(5 \mathrm{~mL})$ was added a solution of $\mathrm{PPh}_{3}(1.074 \mathrm{~g}, 4.1 \mathrm{mmol})$ in DCM (2 $\mathrm{mL}$ ) at $0{ }^{\circ} \mathrm{C}$. The mixture was stirred for $30 \mathrm{~min}$, warmed to $\mathrm{rt}$, and quenched with $\mathrm{NaHCO}_{3}(5 \mathrm{~mL})$. The mixture was extracted with $\mathrm{Et}_{2} \mathrm{O}(15 \mathrm{~mL})$ and EtOAc $(15 \mathrm{~mL})$, washed with brine, and dried over $\mathrm{Na}_{2} \mathrm{SO}_{4}$. The crude material was purified by flash chromatography to afford $\mathbf{2 8 c}$ as a slightly yellow oil $(0.480 \mathrm{~g}, 81 \%)$. IR (neat, $\left.\mathrm{cm}^{-1}\right)$ : $2879,1577,1513,1464,1335,1293,1236,1118,1082 .{ }^{1} \mathrm{H}$ NMR $\left(500 \mathrm{MHz}, \mathrm{CDCl}_{3}\right) \delta$ $8.16(1 \mathrm{H}, \mathrm{d}, J=9.2 \mathrm{~Hz}), 7.79(1 \mathrm{H}, \mathrm{s}), 7.01(1 \mathrm{H}, \mathrm{d}, J=2.8 \mathrm{~Hz}), 6.97(1 \mathrm{H}, J=9.2,2.8 \mathrm{~Hz})$, $4.19(2 \mathrm{H}, \mathrm{t}, J=5.9 \mathrm{~Hz}), 3.51(2 \mathrm{H}, \mathrm{t}, J=5.6 \mathrm{~Hz}), 3.36(3 \mathrm{H}, \mathrm{s}), 2.92(2 \mathrm{H}, \mathrm{t}, J=5.8 \mathrm{~Hz})$, $2.71(2 \mathrm{H}, \mathrm{t}, J=5.6 \mathrm{~Hz}), 2.41(3 \mathrm{H}, \mathrm{s}) .{ }^{13} \mathrm{C} \mathrm{NMR}\left(125 \mathrm{MHz}, \mathrm{CDCl}_{3}\right) \delta 162.8,139.9,135.0$, 134.1, 127.7, 117.0, 115.2, 92.8, 70.7, 67.5, 59.2, 57.6, 56.4, 43.5. HRMS (ESI) calc'd for $\mathrm{C}_{14} \mathrm{H}_{19} \mathrm{~N}_{2} \mathrm{O}_{4} \mathrm{Br}_{2}\left([\mathrm{M}+\mathrm{H}]^{+}\right)$436.9706. Found: 436.9717.

\section{2-(2,2-Dibromovinyl)-4-\{2-[(2-methoxyethyl)-methylamino]-ethoxy\}-phenylamine} (29c) 
<smiles>COCCN(C)CCOc1ccc([N+](=O)[O-])c(C=C(Br)Br)c1</smiles>

A mixture of the nitrobenzene 28c $(0.465 \mathrm{~g}, 1.06 \mathrm{mmol})$ and 1\% Pt-C[V] $(50 \mathrm{mg})$ in $\mathrm{MeOH}(5 \mathrm{~mL})$ was hydrogenated at 25 psi for $4 \mathrm{~h}$ using a Parr hydrogenator. After removal of the catalyst and solvent, the compound was purified by flash chromatography to give 29c as a colorless oil $\left(0.380 \mathrm{~g}, 88 \%\right.$ ). IR (neat, $\left.\mathrm{cm}^{-1}\right)$ : 3437, 3349, 3222, 2874, 1610, 1496, 1259, 1227, 1166, 1117, 1044. ${ }^{1} \mathrm{H}$ NMR (400 MHz, $\left.\mathrm{CDCl}_{3}\right) \delta 7.33(1 \mathrm{H}, \mathrm{s})$, $6.91(1 \mathrm{H}, \mathrm{d}, J=2.7 \mathrm{~Hz}), 6.77(1 \mathrm{H}, \mathrm{dd}, J=8.6,3.0 \mathrm{~Hz}), 6.63(1 \mathrm{H}, \mathrm{d}, J=8.6 \mathrm{~Hz}), 4.02$ $(2 \mathrm{H}, \mathrm{t}, J=6.0 \mathrm{~Hz}), 3.50(2 \mathrm{H}, \mathrm{t}, J=5.7 \mathrm{~Hz}), 3.45(2 \mathrm{H}, \mathrm{br}), 3.35(3 \mathrm{H}, \mathrm{s}), 2.83(2 \mathrm{H}, \mathrm{t}, J=$ $6.0 \mathrm{~Hz}), 2.69(2 \mathrm{H}, \mathrm{t}, J=5.8 \mathrm{~Hz}), 2.39(3 \mathrm{H}, \mathrm{s}) .{ }^{13} \mathrm{C} \mathrm{NMR}\left(100 \mathrm{MHz}, \mathrm{CDCl}_{3}\right) \delta 151.7$, $137.7,134.1,122.8,117.4,117.2,114.9,92.7,70.8,67.0,59.0,57.4,56.8,43.5$. HRMS calc'd for $\mathrm{C}_{14} \mathrm{H}_{21} \mathrm{~N}_{2} \mathrm{O}_{2} \mathrm{Br}_{2}\left([\mathrm{M}+\mathrm{H}]^{+}\right)$406.9964. Found: 406.9977.

\section{(2-Methoxyethyl)-\{2-[2-(2-methoxyquinolin-3-yl)-1H-indol-5-yloxy]-ethyl\}- methylamine (30c)}

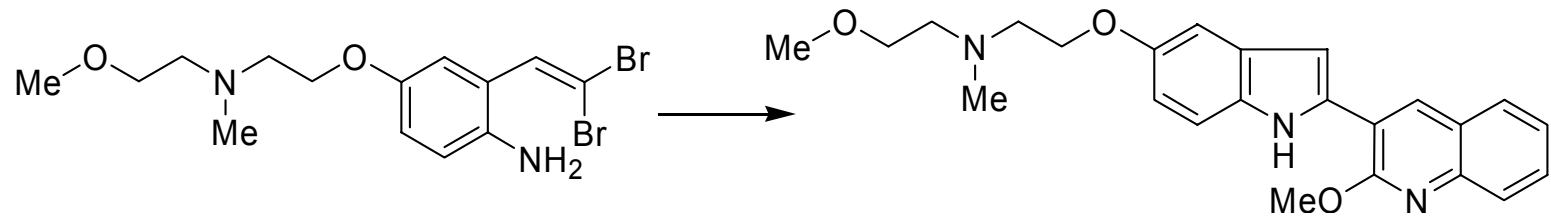

To a $5 \mathrm{~mL}$ round-bottomed flask was charged with aniline $29 \mathrm{c}(0.122 \mathrm{~g}, 0.3 \mathrm{mmol})$, boronic acid 13 (0.0914 g, $0.45 \mathrm{mmol}), \mathrm{Pd}(\mathrm{OAc})_{2}(3.4 \mathrm{mg}, 0.015 \mathrm{mmol})$, S-Phos (12.3 $\mathrm{mg}, 0.03 \mathrm{mmol})$, and $\mathrm{K}_{3} \mathrm{PO}_{4} \cdot \mathrm{H}_{2} \mathrm{O}(0.345 \mathrm{~g}, 1.5 \mathrm{mmol})$. After the flask was purged with argon for $10 \mathrm{~min}$, PhMe $(1.5 \mathrm{~mL})$ was added was and the reaction mixture was heated at $100{ }^{\circ} \mathrm{C}$ for $2 \mathrm{~h}$. The reaction mixture was then quenched with $\mathrm{NaHCO}_{3}$, extracted with EtOAc $(3 \times 10 \mathrm{~mL})$, and dried over $\mathrm{Na}_{2} \mathrm{SO}_{4}$. The crude material was purifed by flash chromatography using 33\% EtOAc in hexanes containing 3\% $i \mathrm{PrNH}_{2}$ to afford the product 30c as a slightly yellow oil $(0.0832 \mathrm{~g}, 68 \%)$. IR (neat, $\left.\mathrm{cm}^{-1}\right): 1456,2943,1622$, 1475, 1398, 1194, 1118. ${ }^{1} \mathrm{H}$ NMR (400 MHz, $\left.\mathrm{CDCl}_{3}\right) \delta 9.54(1 \mathrm{H}, \mathrm{br}), 8.41(1 \mathrm{H}, \mathrm{s}), 7.84$ $(1 \mathrm{H}, \mathrm{d}, J=8.2 \mathrm{~Hz}), 7.75(1 \mathrm{H}, \mathrm{dd}, J=8.0,1.1 \mathrm{~Hz}), 7.60(1 \mathrm{H}, \mathrm{ddd}, J=8.3,7.1,1.4 \mathrm{~Hz})$, 
$7.40(1 \mathrm{H}, \mathrm{ddd}, J=7.5,7.5,1.0 \mathrm{~Hz}), 7.32(1 \mathrm{H}, \mathrm{d}, J=8.8 \mathrm{~Hz}), 7.10(1 \mathrm{H}, \mathrm{d}, J=2.3 \mathrm{~Hz})$, $6.98(1 \mathrm{H}, \mathrm{dd}, J=1.6 \mathrm{~Hz}), 6.89(1 \mathrm{H}, \mathrm{dd}, J=8.8,2.3 \mathrm{~Hz}), 4.26(3 \mathrm{H}, \mathrm{s}), 4.16(2 \mathrm{H}, \mathrm{t}, J=6.0$ $\mathrm{Hz}), 3.53(2 \mathrm{H}, \mathrm{t}, J=5.7 \mathrm{~Hz}), 3.37(3 \mathrm{H}, \mathrm{s}), 2.91(2 \mathrm{H}, \mathrm{t}, J=6.0 \mathrm{~Hz}), 2.73(2 \mathrm{H}, \mathrm{t}, J=5.6$ $\mathrm{Hz}), 2.43(3 \mathrm{H}, \mathrm{s}) .{ }^{13} \mathrm{C}$ NMR $\left(100 \mathrm{MHz}, \mathrm{CDCl}_{3}\right) \delta 158.4,153.8,145.3,135.1,134.2$, $131.9,129.6,128.8,127.6,127.1,125.7,125.0,116.9,113.9,112.1,103.0,101.1,70.9$, 66.9, 59.1, 57.4, 57.0, 54.2, 43.5. HRMS calc'd for $\mathrm{C}_{24} \mathrm{H}_{28} \mathrm{~N}_{3} \mathrm{O}_{3}\left([\mathrm{M}+\mathrm{H}]^{+}\right) 406.2125$. Found: 406.2130 .

\section{3-(5-\{2-[(2-Methoxyethyl)-methylamino]-ethoxy\}-1H-indol-2-yl)-1H-quinolin-2-one}

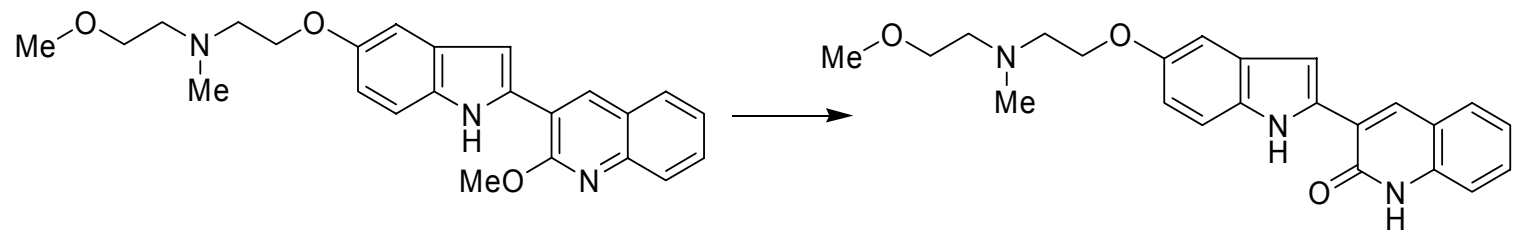

A mixture of 30c $(41 \mathrm{mg}, 0.101 \mathrm{mmol})$ and $\mathrm{HCl}(2 \mathrm{~mL}, 3 \mathrm{M})$ was heated to $90{ }^{\circ} \mathrm{C}$ overnight. Solid $\mathrm{K}_{2} \mathrm{CO}_{3}$ was carefully added to the mixture until it is neutral, and the mixture was filtered through, and the collected product was washed with $\mathrm{H}_{2} \mathrm{O}$ and dried under vacuum to afford 4 as a yellow solid (35.4 mg, 90\%). ${ }^{1} \mathrm{H}$ NMR (400 $\mathrm{MHz}, \mathrm{CDCl}_{3}$ ) $\delta 11.32(1 \mathrm{H}, \mathrm{br}), 11.07(1 \mathrm{H}, \mathrm{s}), 8.27(1 \mathrm{H}, \mathrm{s}), 7.61(1 \mathrm{H}, \mathrm{d}, J=7.6 \mathrm{~Hz}), 7.46$ (1H, ddd, $J=$ 7.7, 7.7, 1.1 Hz), $7.35(1 \mathrm{H}, \mathrm{d}, J=8.8 \mathrm{~Hz}), 7.28-7.23(2 \mathrm{H}, \mathrm{m}), 7.09(1 \mathrm{H}, \mathrm{d}, J=2.2 \mathrm{~Hz})$, $6.94(1 \mathrm{H}, \mathrm{d}, J=1.4 \mathrm{~Hz}), 6.90(1 \mathrm{H}, \mathrm{dd}, J=8.8,2.3 \mathrm{~Hz}, 4.17(2 \mathrm{H}, \mathrm{t}, J=6.1 \mathrm{~Hz}), 3.55(2 \mathrm{H}$, $\mathrm{t}, J=5.7 \mathrm{~Hz}), 3.38(3 \mathrm{H}, \mathrm{s}), 2.93(2 \mathrm{H}, \mathrm{t}, J=6.0 \mathrm{~Hz}), 2.75(2 \mathrm{H}, \mathrm{t}, J=5.8 \mathrm{~Hz}), 2.45(3 \mathrm{H}, \mathrm{s})$.

${ }^{13} \mathrm{C}$ NMR $\left(100 \mathrm{MHz}, \mathrm{CDCl}_{3}\right) \delta 163.1,153.8,136.9,134.8,134.7,131.9,130.5,128.5$, 128.1, 123.6, 122.3, 120.7, 115.5, 114.1, 112.6, 102.7, 99.4, 70.9, 66.9, 59.1, 57.5, 57.0, 43.5. HRMS (ESI) calc'd for $\mathrm{C}_{23} \mathrm{H}_{26} \mathrm{~N}_{3} \mathrm{O}_{2}\left([\mathrm{M}+\mathrm{H}]^{+}\right)$392.1968. Found: 392.1983. 

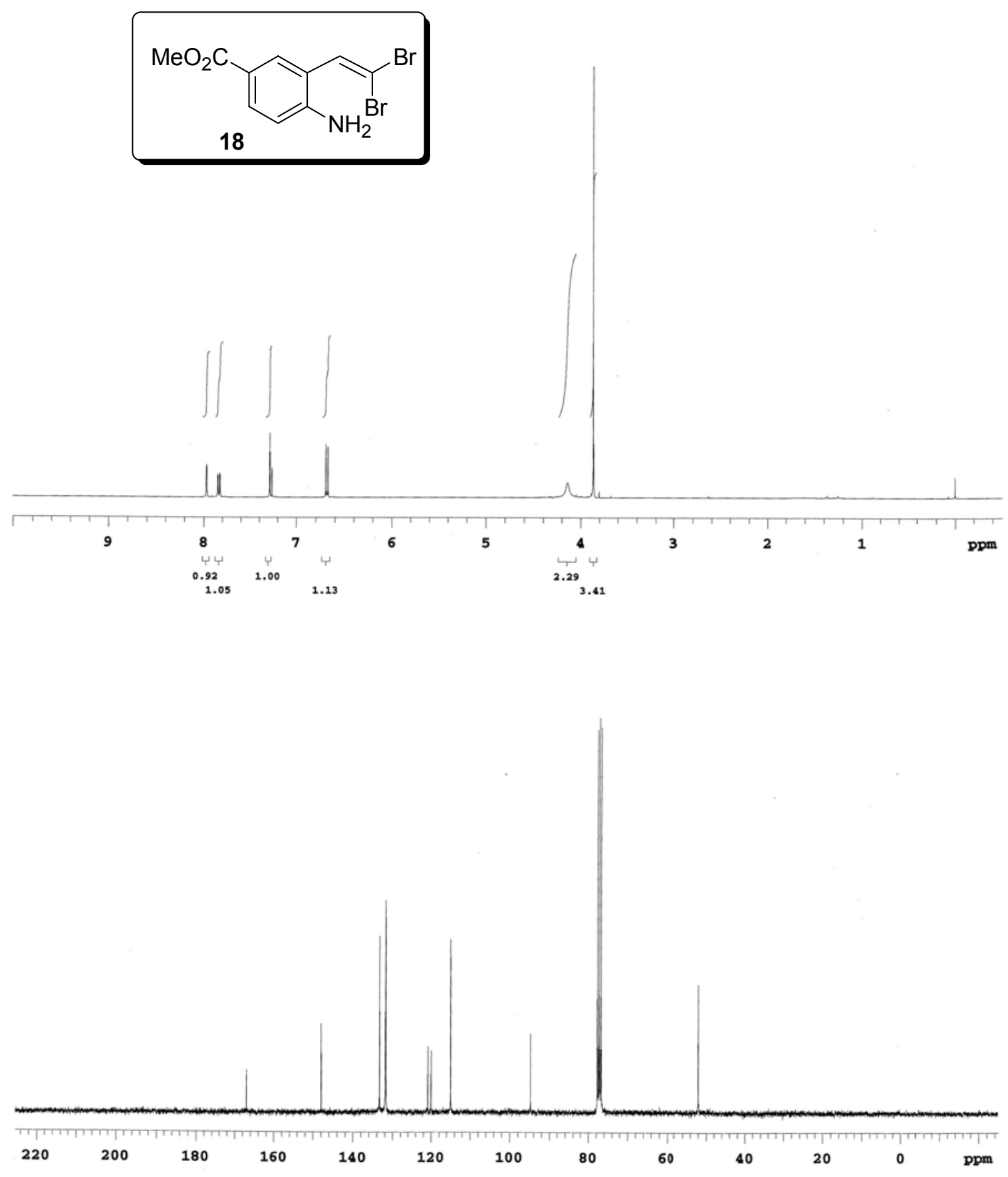
-S10-
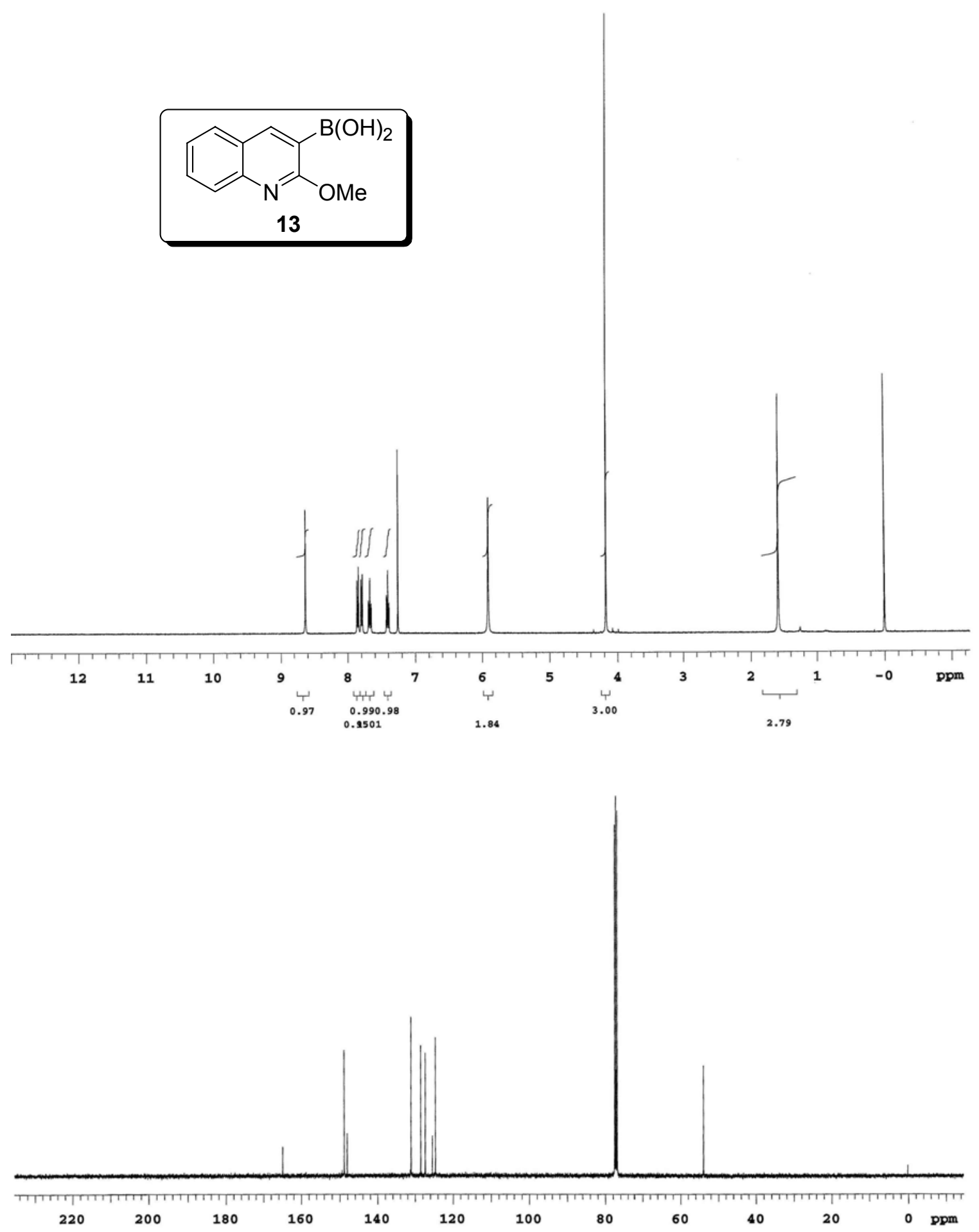
-S11-
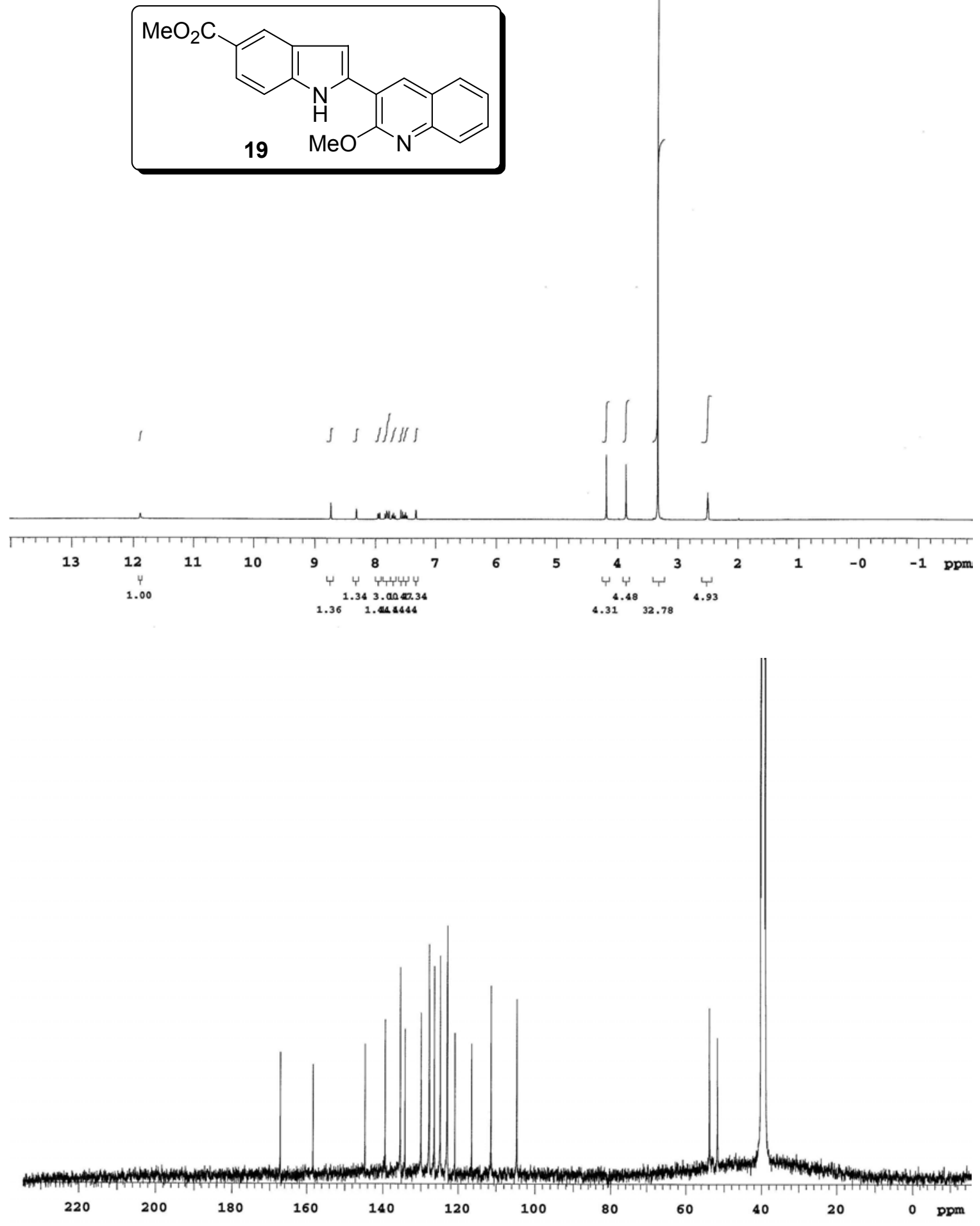

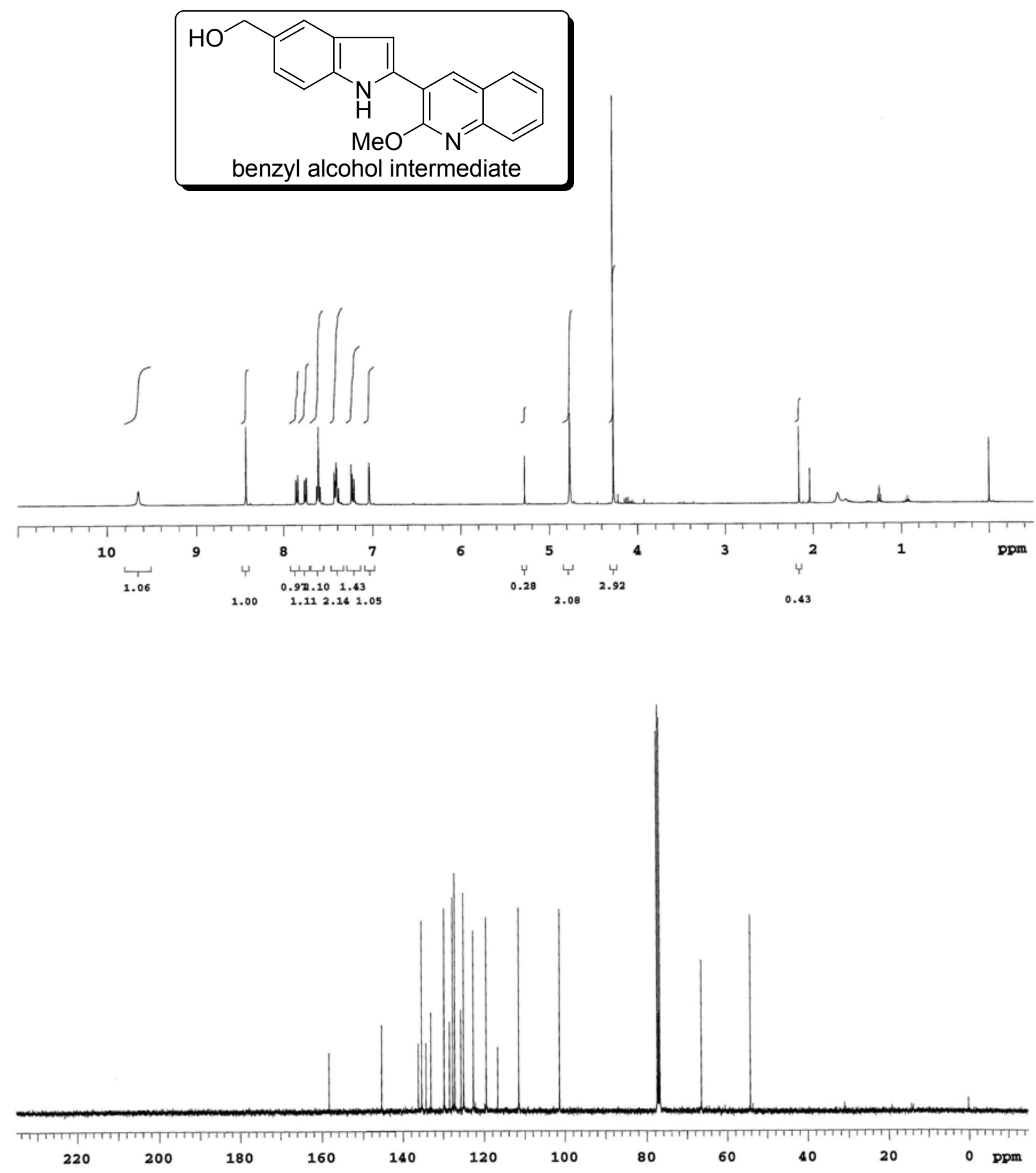

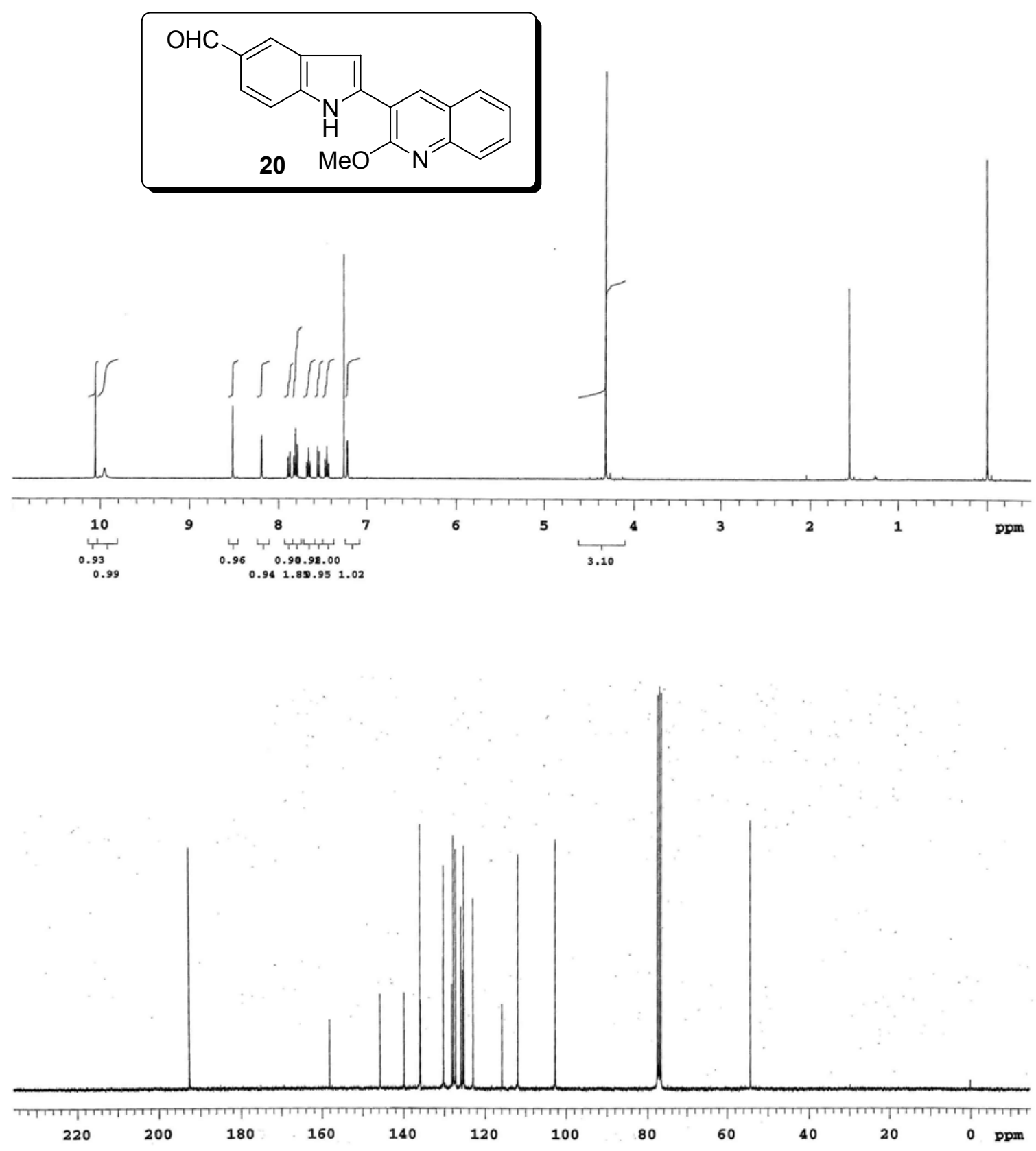
-S14-
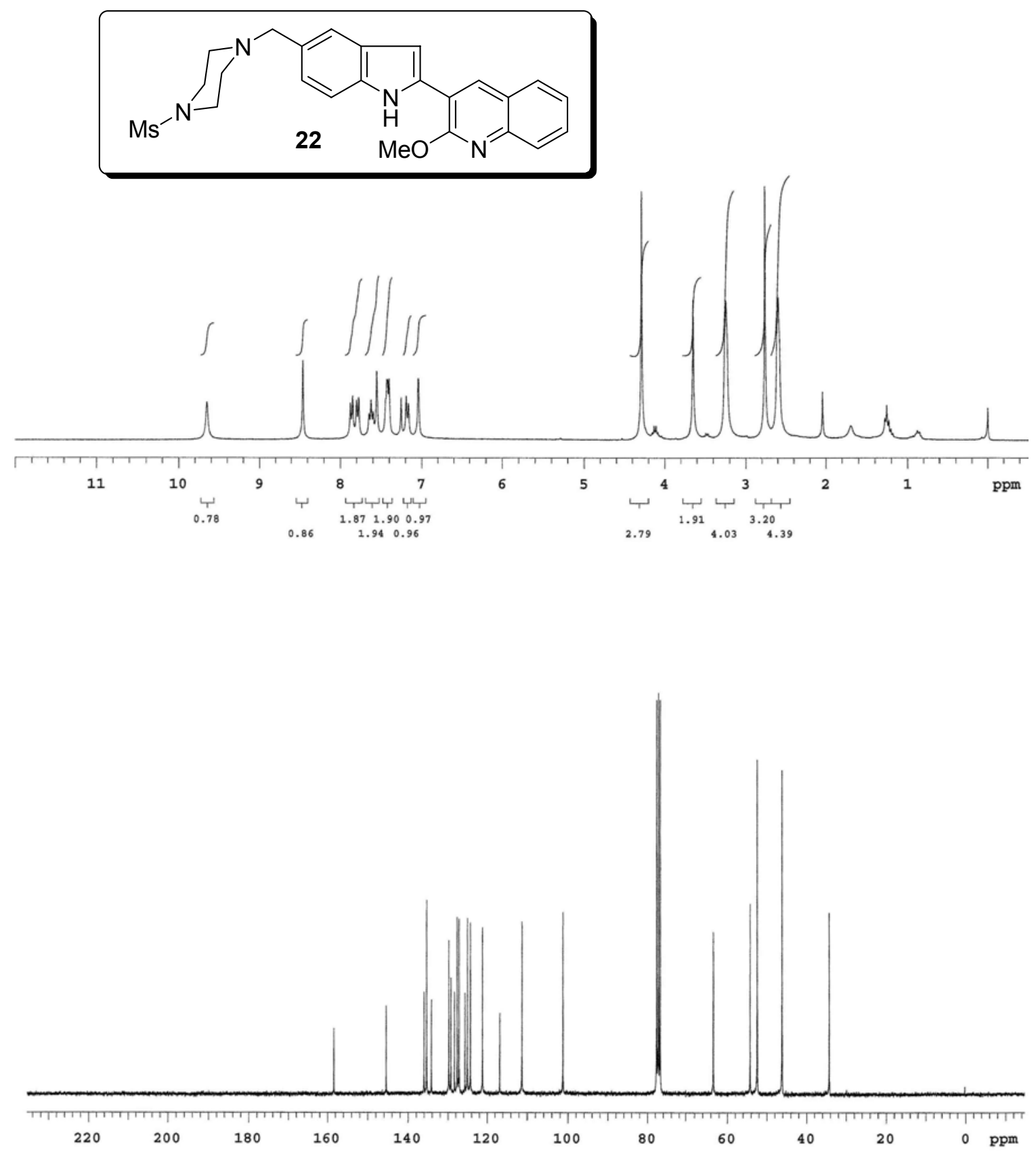
-S15-
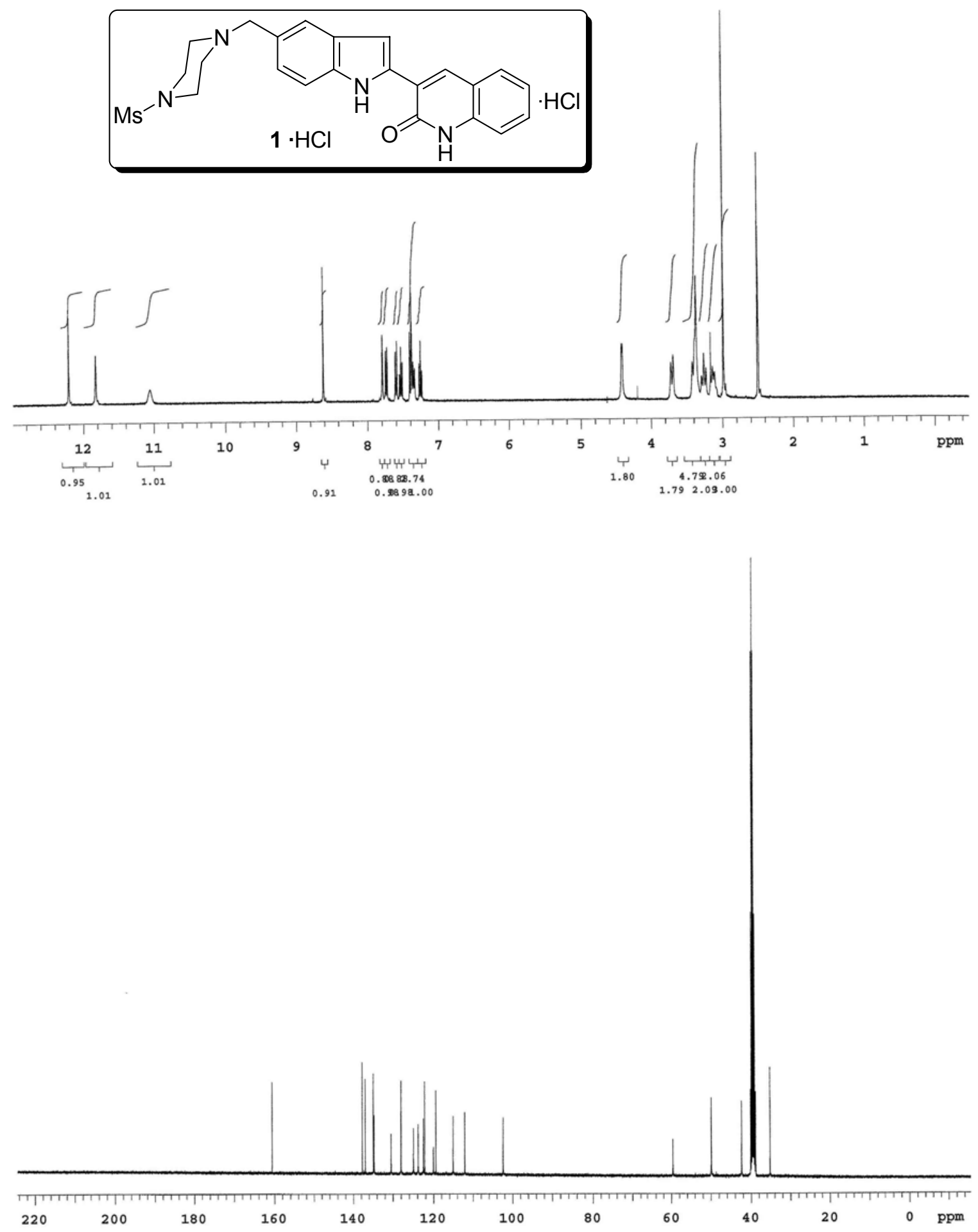

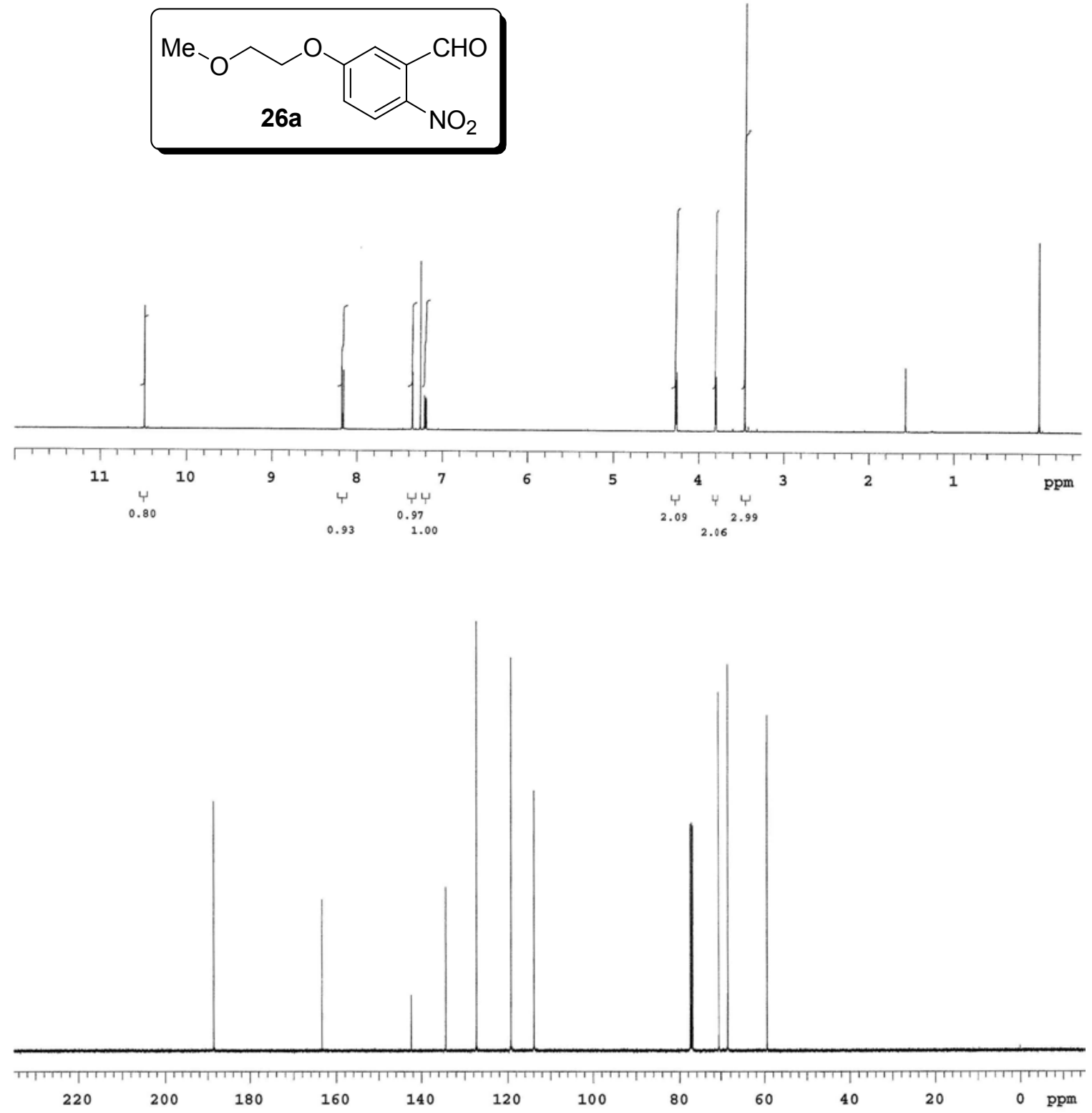

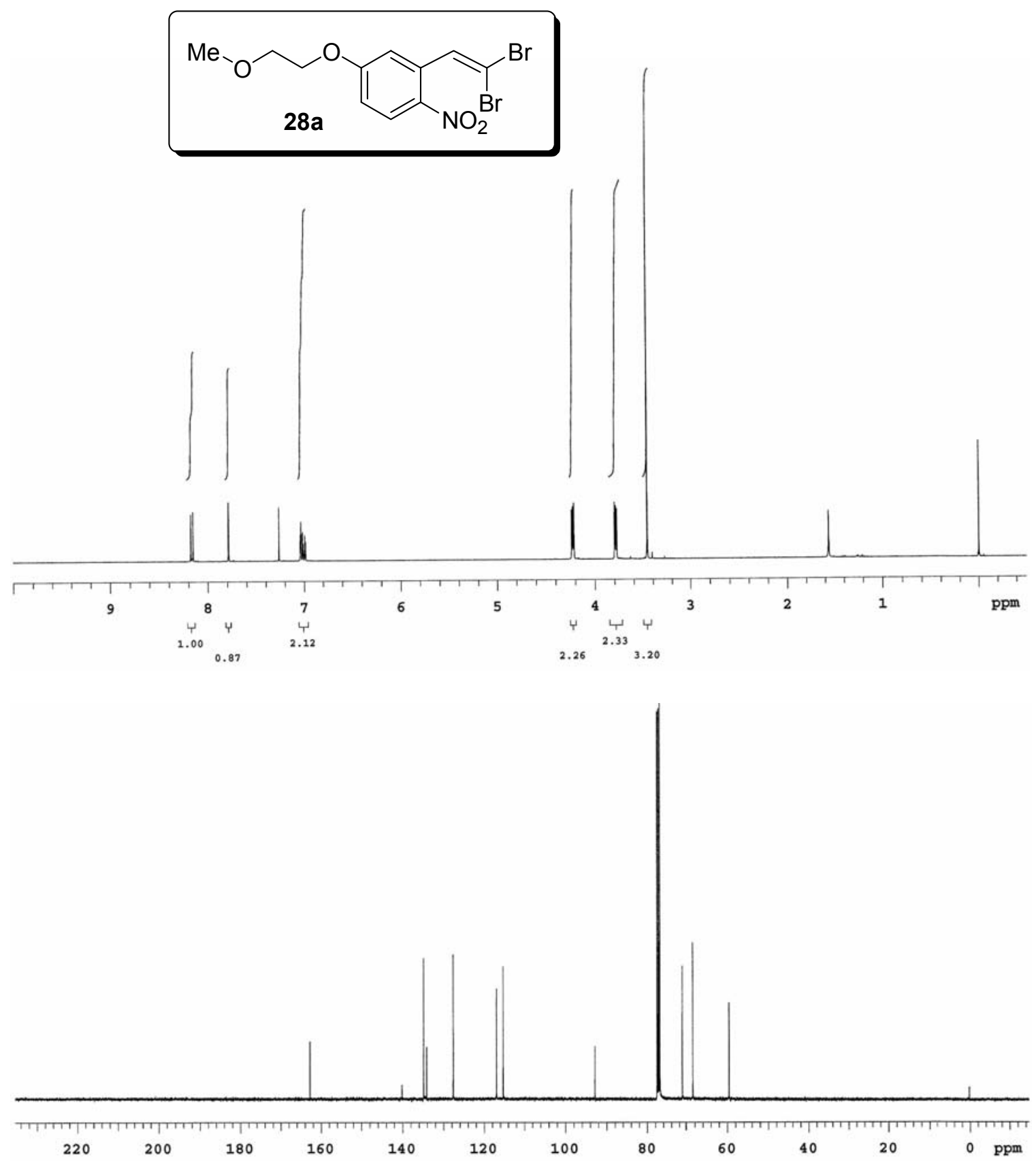
-S18-
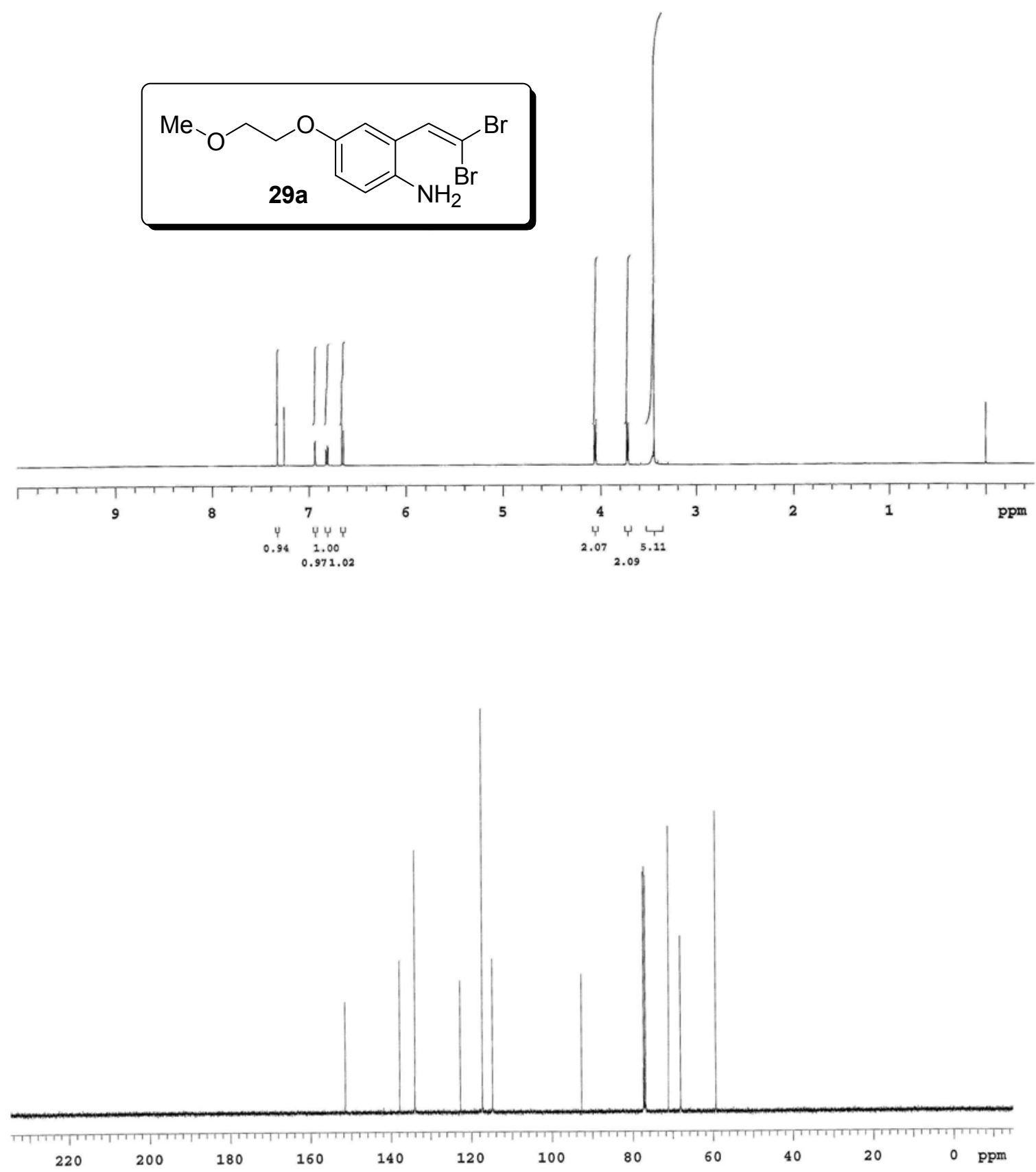

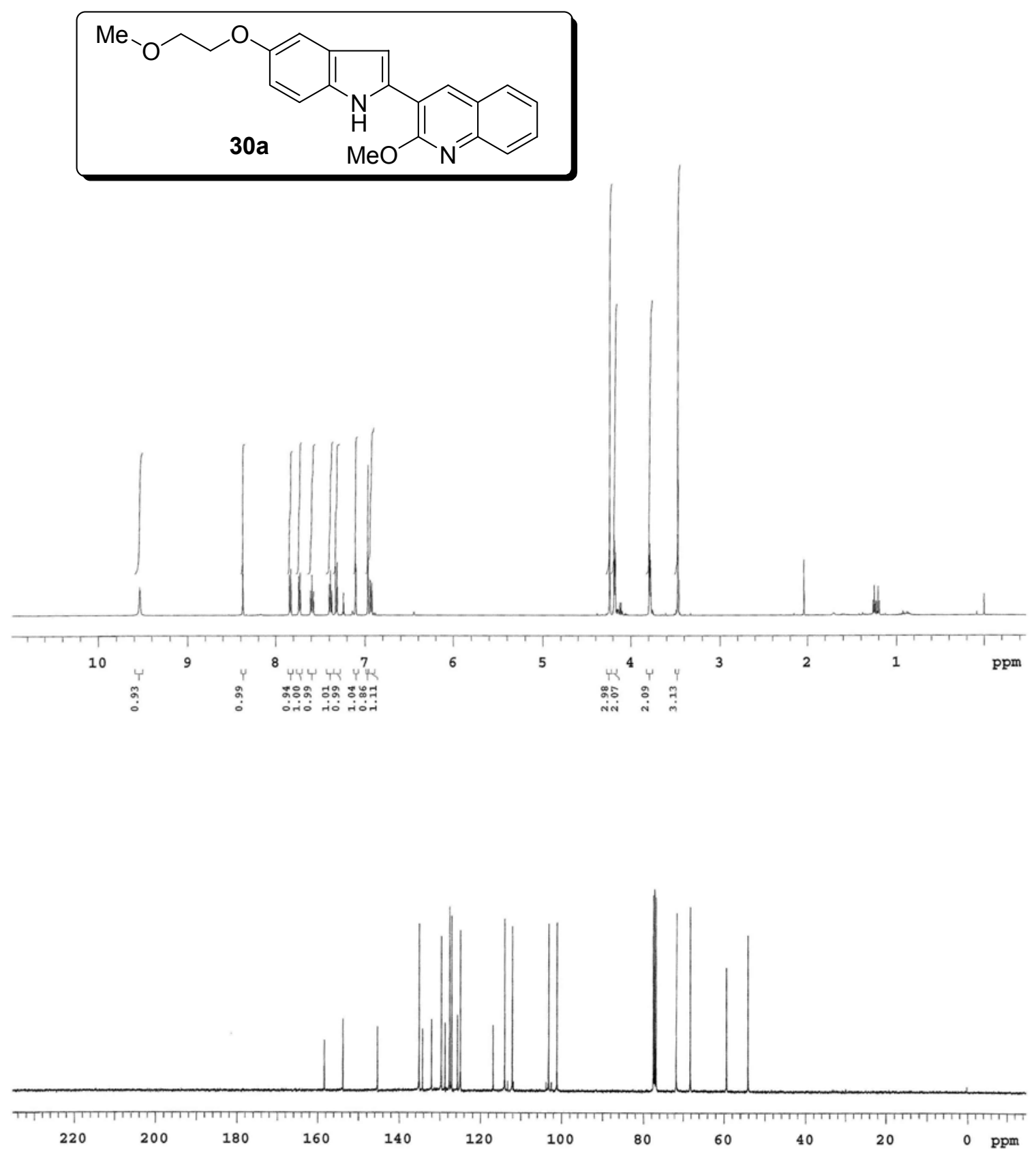

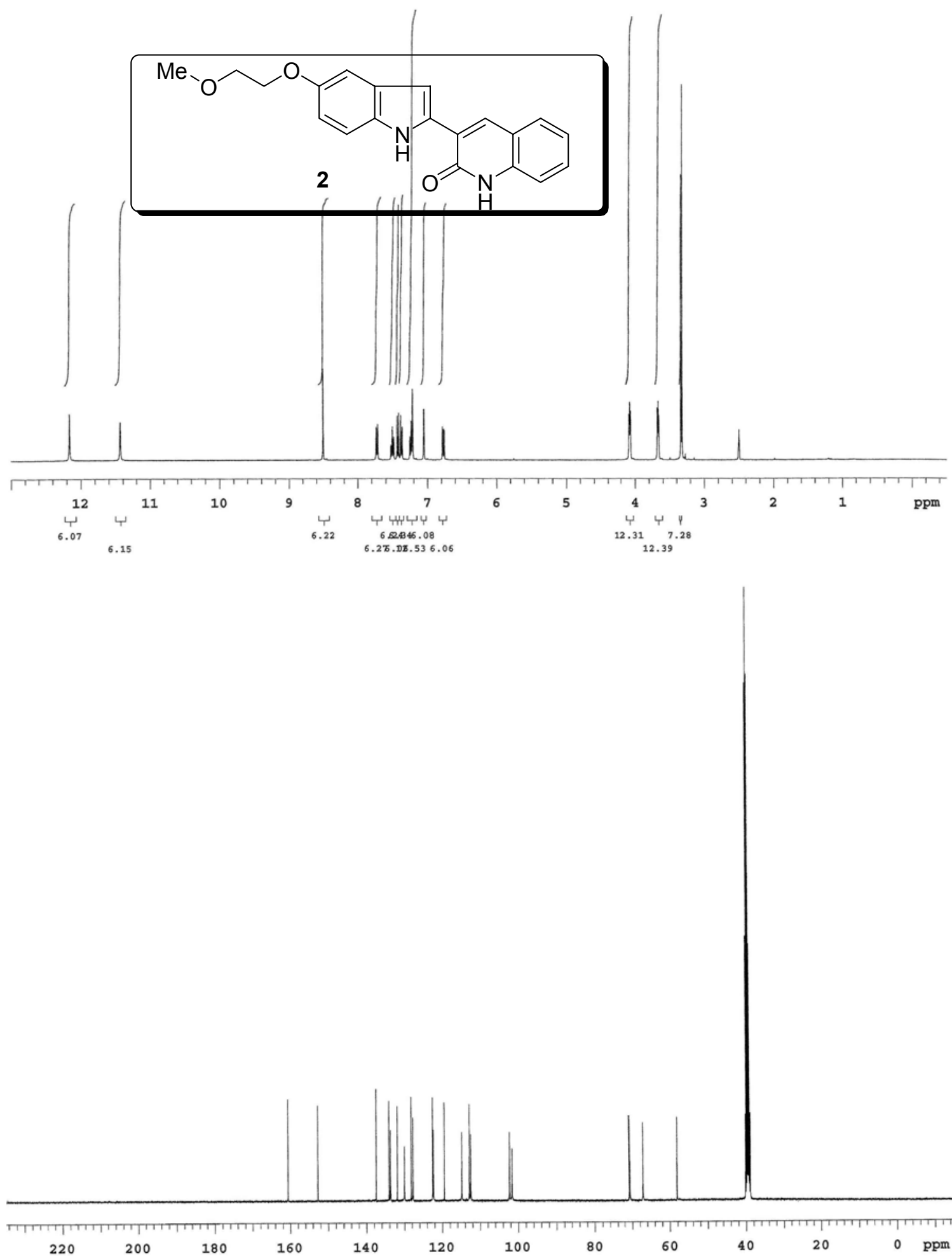

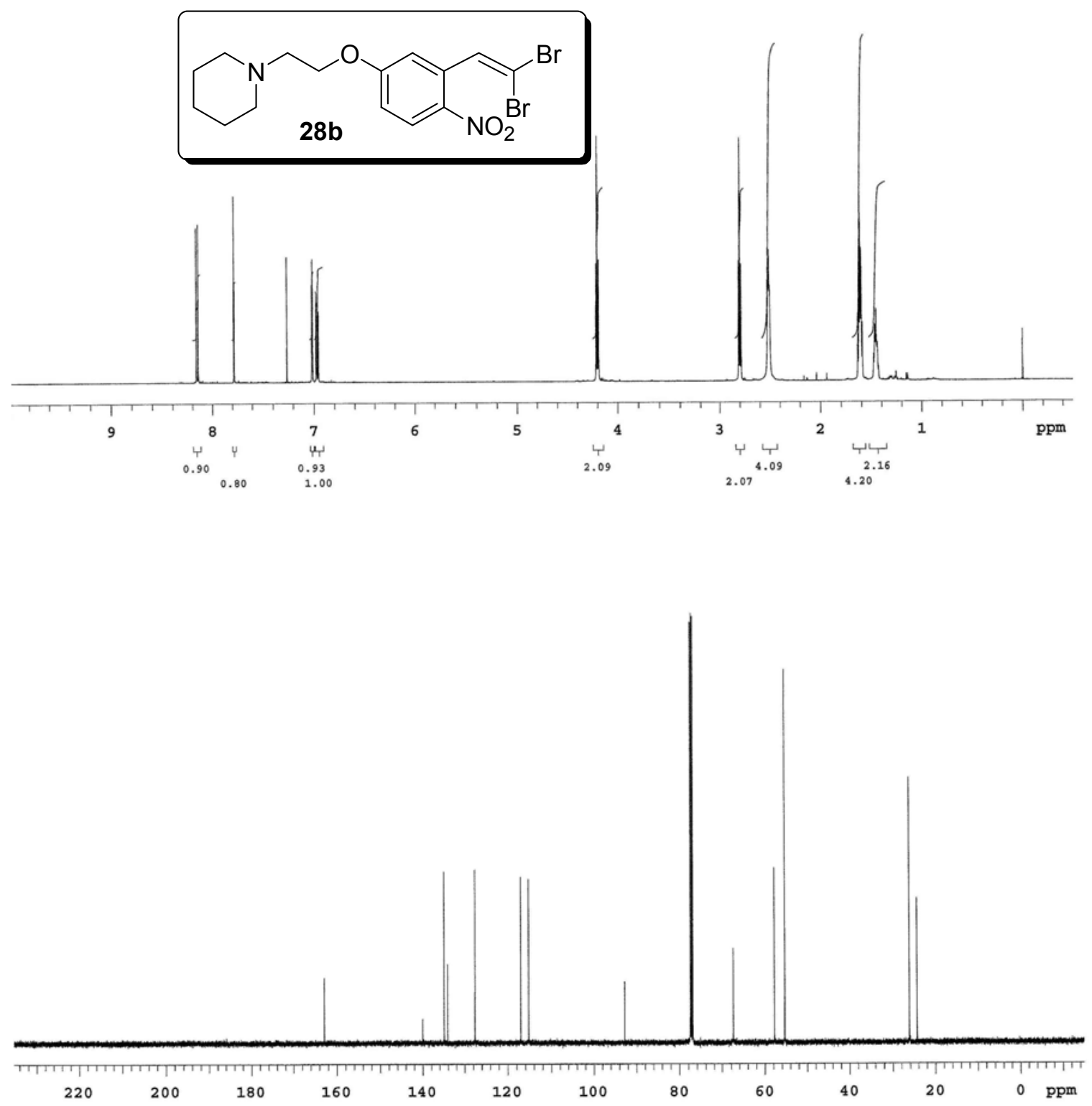

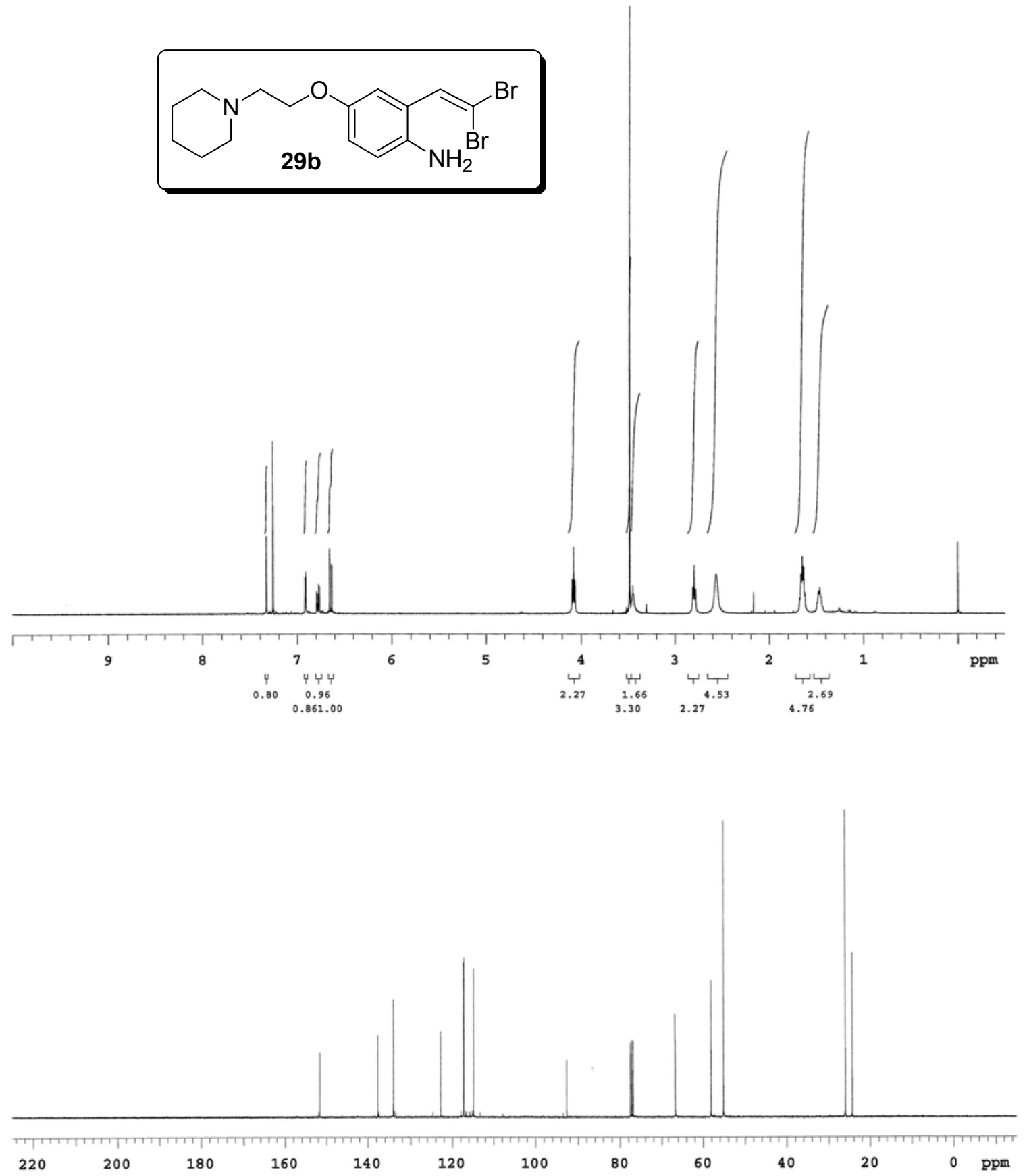

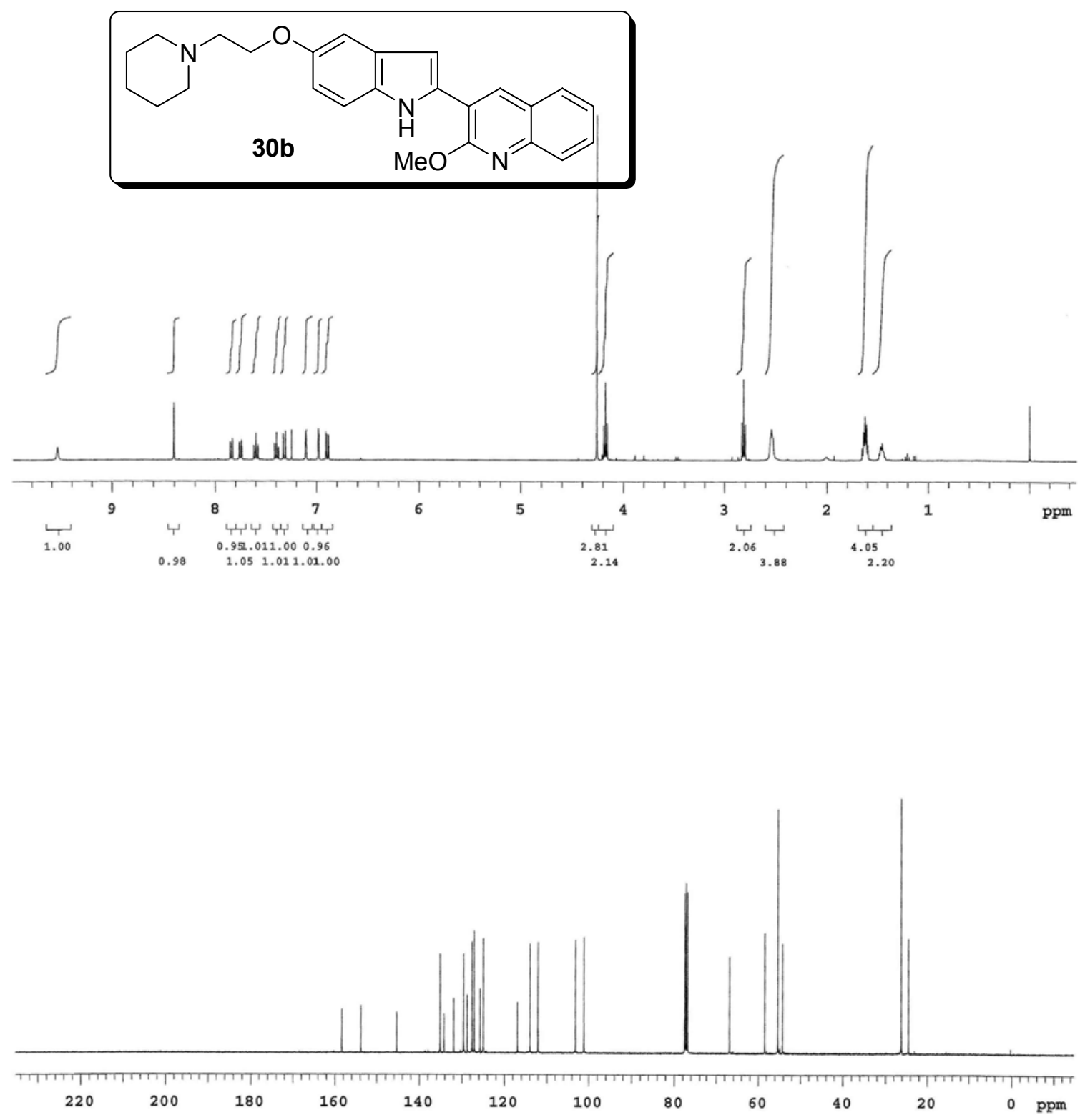

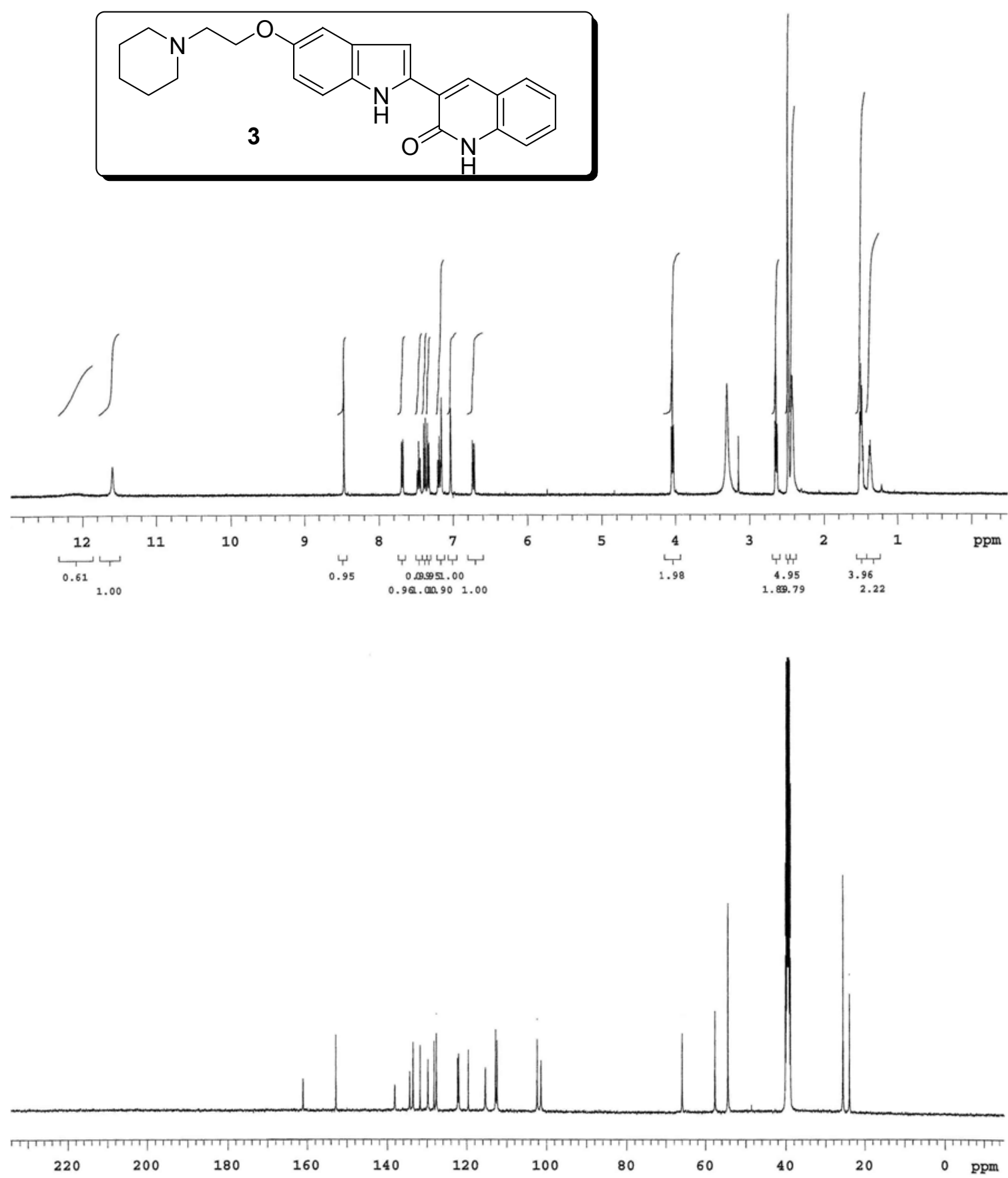

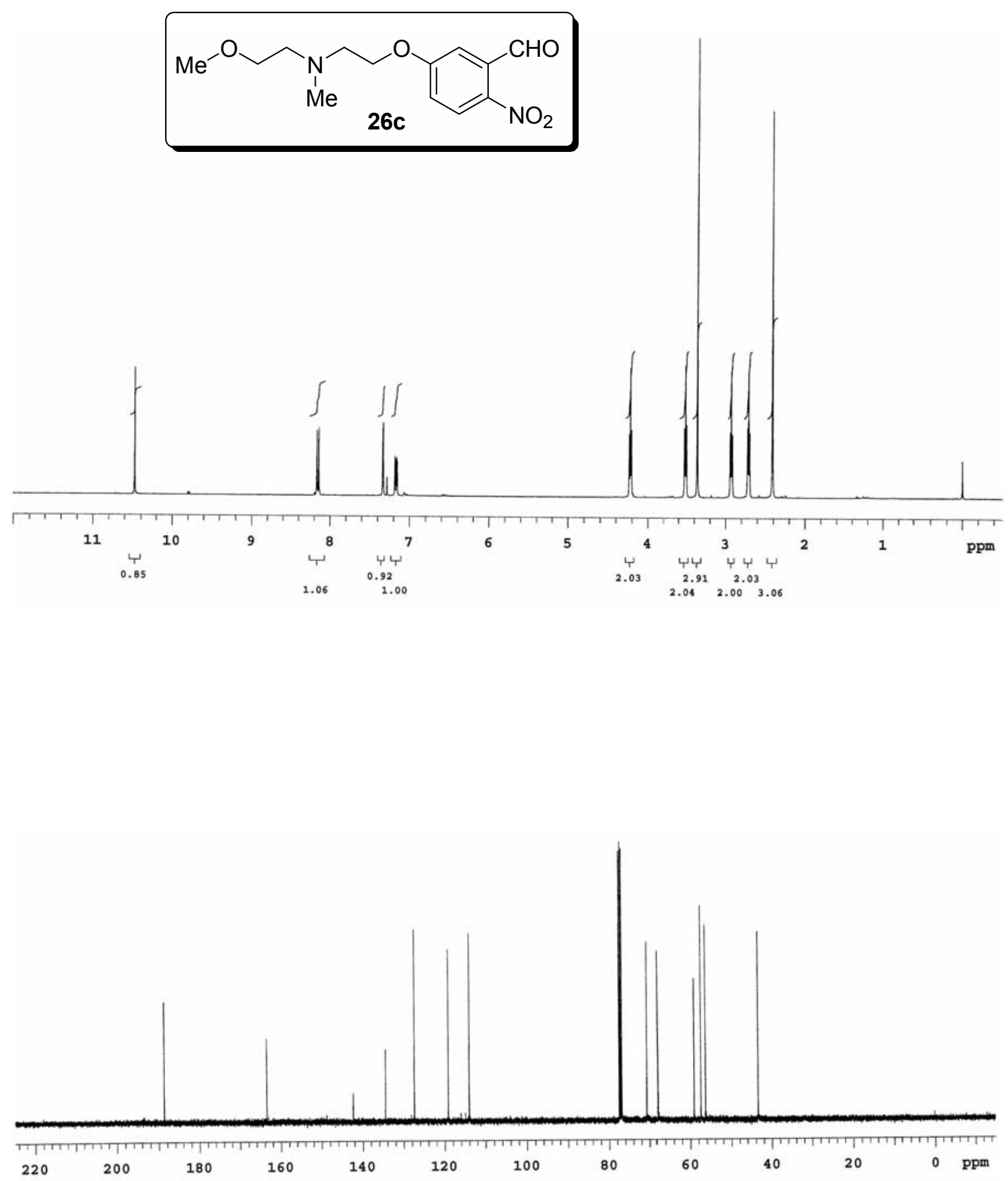

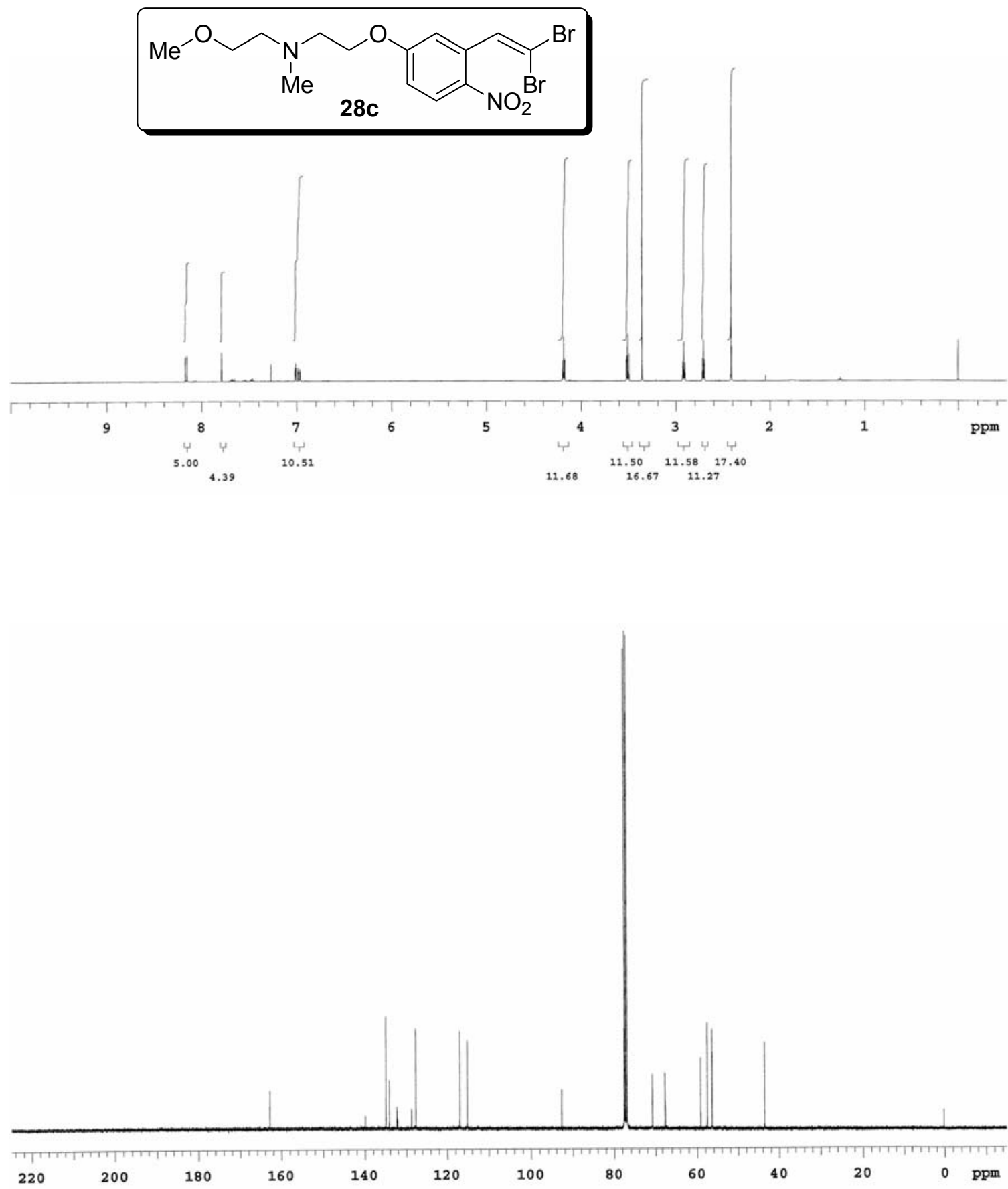

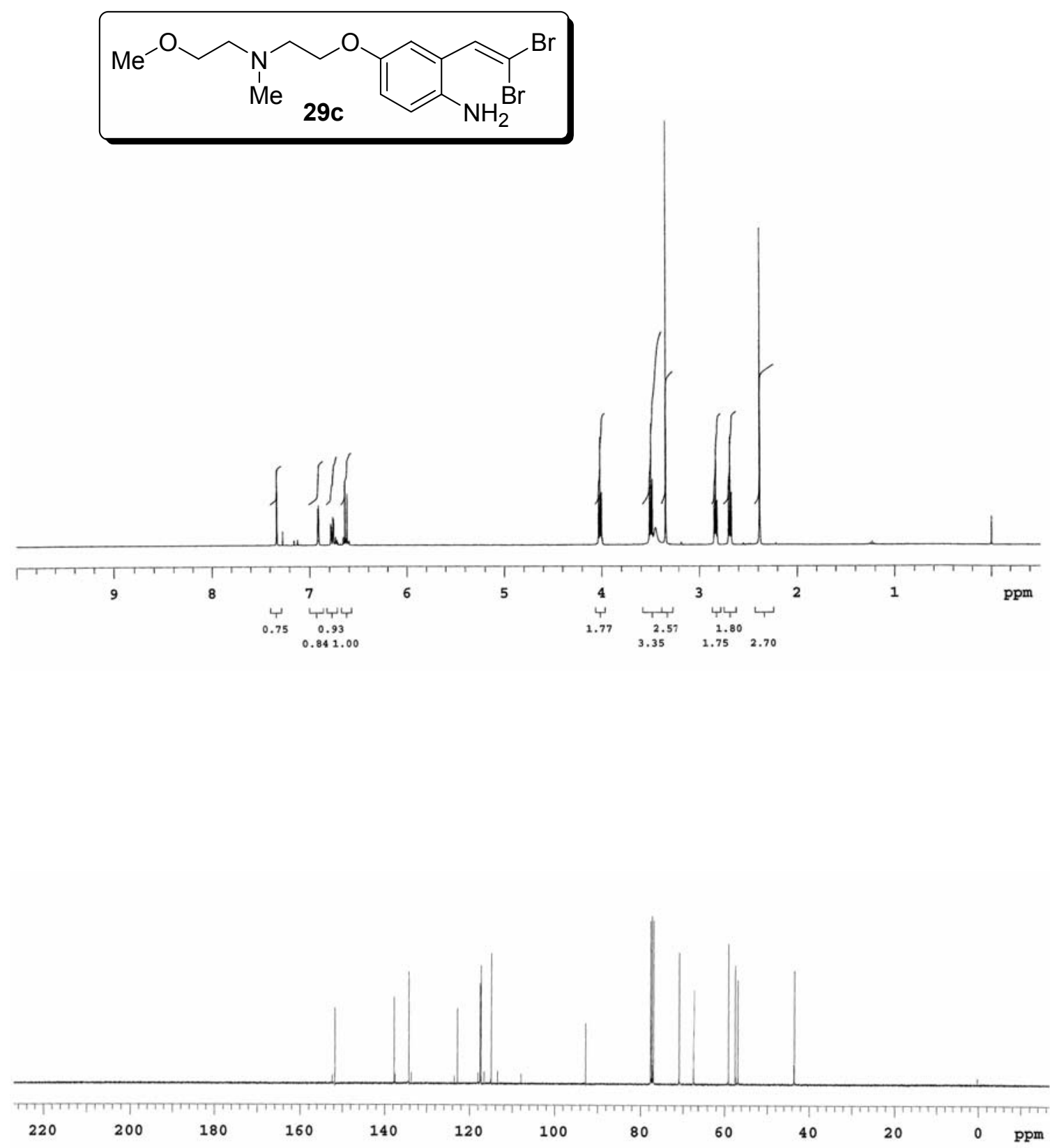

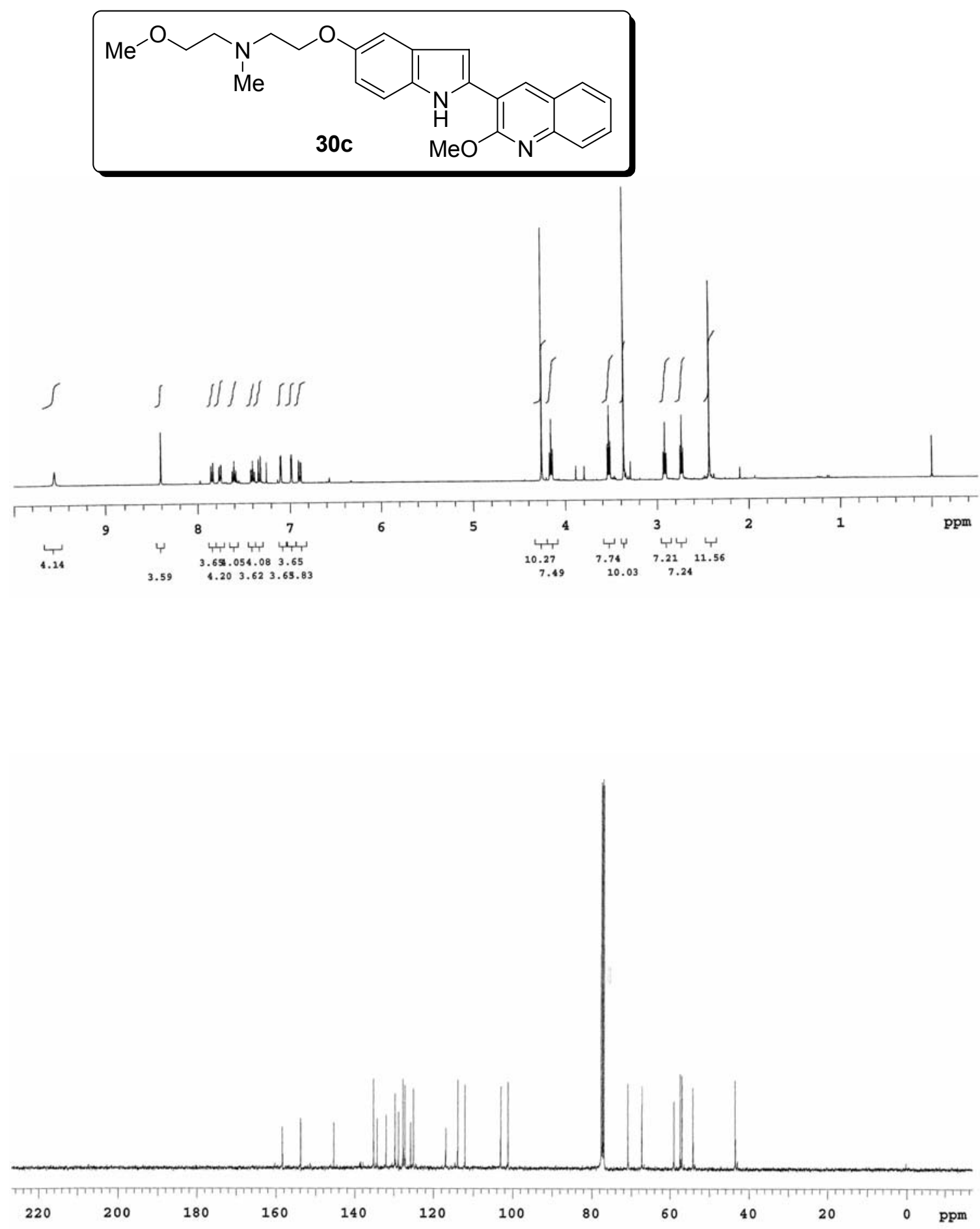

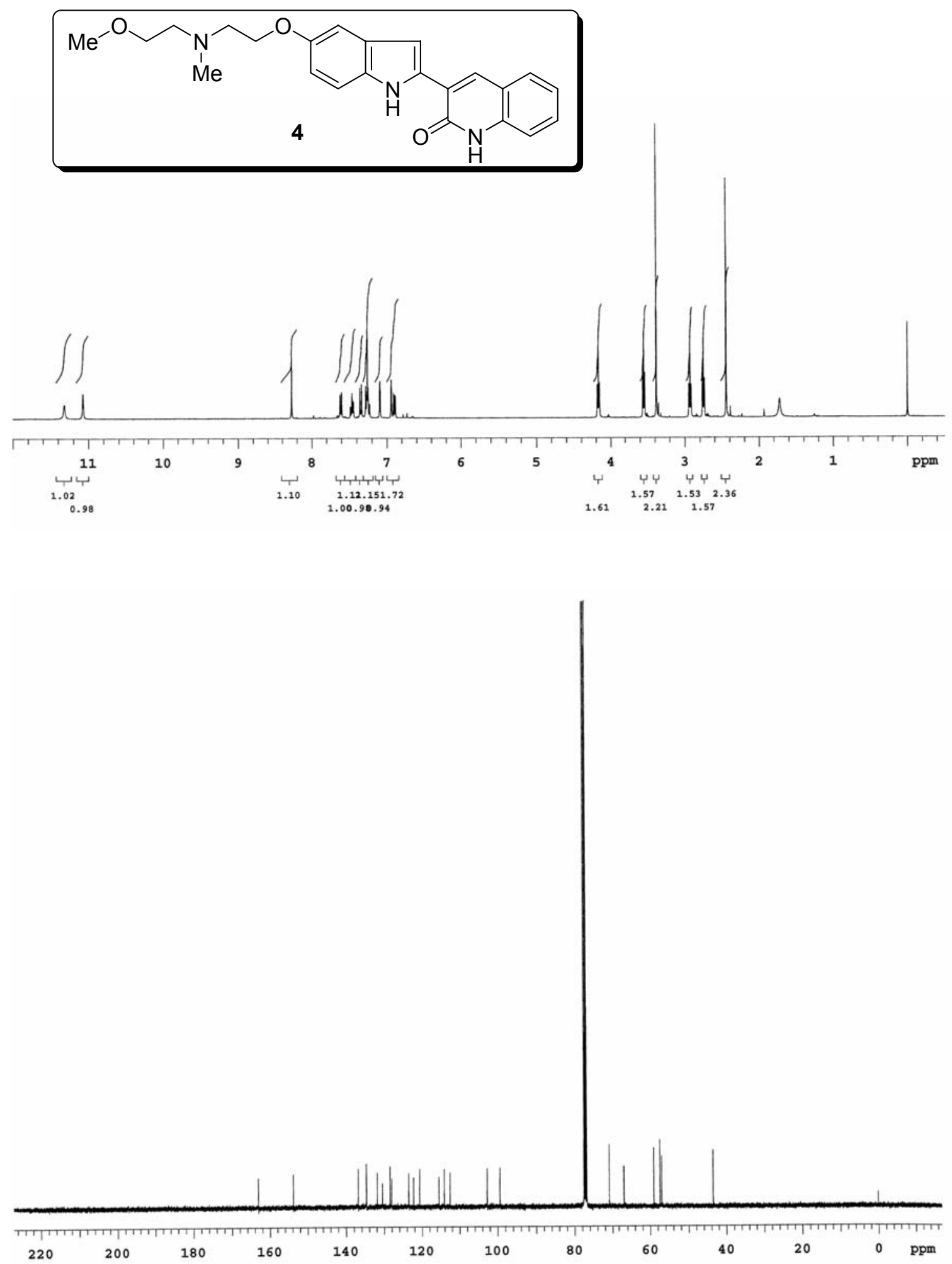